\title{
Wind velocity distribution reconstruction using CFD database with Tucker decomposition and sensor measurement
}

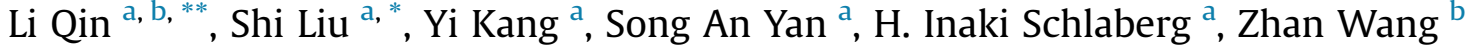 \\ a School of Energy Power \& Mechanical Engineering, North China Electric Power University, Beijing 102206, China \\ ${ }^{\mathrm{b}}$ Key Laboratory for Mechanics in Fluid Solid Coupling Systems, Institute of Mechanics, Chinese Academy of Sciences, Beijing 100190, China
}

\section{A R T I C L E I N F O}

\section{Article history:}

Received 29 May 2018

Received in revised form

4 October 2018

Accepted 5 November 2018

Available online 12 November 2018

\section{Keywords:}

Wind velocity distribution

Third-order CFD database

Tucker decomposition

Sensor measurement

\begin{abstract}
A B S T R A C T
Wind forecasting holds the key to the management of wind power. Previous vector or matrix wind forecast methods may not best reflect the intrinsic inter relationship among the wind velocity components of a three-dimensional wind field. Alternatively, a tensor-based model is developed to reconstruct the wind velocity distribution within a short period of time, enabling a new way for wind forecasting. A third-order CFD database is established by CFD simulations and the Tucker decomposition is used to obtain the tensor basis off site. Then in real time, the tensor basis can be employed to rapidly reconstruct wind velocity distributions in any direction, which can also form a new way to reconstruct wind velocity distribution in 3-D spaces. A comparison of the maximum and relative reconstruction errors shows that the newly proposed method performs better than the authors' previously published wind field reconstruction method. The influences of sampling rate, noise level and sensor distributions on the reconstruction error are also discussed in this paper. Finally, a wind tunnel experiment is carried out to evaluate the accuracy of the proposed method, and in most cases, the experimental results show that relative errors drop around $0.03 \%-0.4 \%$ and maximum errors drop around $0.02 \%-1.7 \%$ when using the newly proposed method.
\end{abstract}

() 2018 Elsevier Ltd. All rights reserved.

\section{Introduction}

Wind energy is the main source of renewable energy. Although the wind resource is abundant, its intermittent and variable characteristics make it difficult to balance a constant supply of electricity. That is why wind velocity forecasting is so important, and many interesting researches have been devoted to solve this problem [1]. There are many forecasting ways which can be broadly divided into two categories, statistical models, numerical weather prediction models (also called physical models) [2]. Physical models, which are mainly based on numerical weather forecasts $[3,4]$ usually take various meteorological data (such as wind direction, temperature, humidity, air density) into consideration. Statistical models [5], such as time-series methods [6,7], Artificial Neural Networks methods [8], Kalman Filter methods [9] and Support Vector Machine methods [10], are mainly used to find the relationship between the input data and the output wind speed or

\footnotetext{
* Corresponding author.

** Corresponding author. School of Energy Power \& Mechanical Engineering, North China Electric Power University, Beijing 102206, China

E-mail addresses: qinli711@126.com (L. Qin), liushi@ncepu.edu.cn (S. Liu).
}

wind power, using historical wind data. Yang et al. proposed a hybrid forecasting method to forecast wind speed [11], while it is also based on historical data. In Ref. [12], Dong et al. proposed a day-ahead prediction model based on NWP data and wind power data from a wind farm, but this method only focuses on the forecast in specific positions, not a whole wind field. Khosravi [6] et al. proposed a time-series wind prediction method employing machine learning methods that can predict future values of the system based on past data, but this method cannot predict the wind velocity distribution in a continuous space.

In order to predict the wind velocity distribution in a continuous space, Qin et al. [13] introduced a wind field reconstruction method based on PCA and CFD data, which can predict wind velocity distributions in a short time. However, this method is essentially a vector or matrix-based method that in the current cases very often treats the wind velocity components of a three-dimensional (3D) wind field as separate entities, which may destroy their intrinsic inner relationship. Therefore, methods that can overcome this shortcoming need to be developed. Compared with vector and matrix-based methods, tensor-based methods usually have better performance in representing high-dimensional data. Higher-Order Singular Value Decomposition (HOSVD) which is also called the 


\begin{tabular}{|c|c|c|c|}
\hline \multicolumn{2}{|c|}{ Nomenclature } & $\mathrm{R}$ & Domain of analysis \\
\hline A & Location-based matrix & $\rho$ & Density (kg/m^3) \\
\hline B & Co-efficient matrix & $\mathrm{p}$ & Pressure $(\mathrm{Pa})$ \\
\hline$S$ & Core tensor & \multicolumn{2}{|c|}{ Subscripts } \\
\hline $\mathrm{U}^{(1)}$ & Mode-1 factorization factor matrix & i & Nodal point \\
\hline $\mathrm{U}^{(2)}$ & Mode-2 factorization factor matrix & $\mathrm{n}, \mathrm{k}$ & Position of elements in a matrix \\
\hline $\mathrm{U}^{(3)}$ & Mode-3 factorization factor matrix & $\mathrm{m}$ & Number of samples \\
\hline $\mathrm{N}$ & Normal distribution function & & \\
\hline $\mathrm{n}$ & Number of data points & \multicolumn{2}{|c|}{ Abbreviations } \\
\hline V & CFD database (Sample tensor) & ANSYS & Analysis Systems \\
\hline W & Basis tensor & CFD & Computational Fluid Dynamics \\
\hline $\mathrm{X}$ & Original wind field matrix $(\mathrm{m} / \mathrm{s})$ & MATLAB & Matrix Laboratory \\
\hline $\mathrm{X}_{\mathrm{R}}$ & Reconstructed wind filed matrix $(\mathrm{m} / \mathrm{s})$ & PCA & Principal Component Analysis \\
\hline Y & Measured data in the domain $(\mathrm{m} / \mathrm{s})$ & HOSVD & Higher-Order Singular Value Decomposition \\
\hline$\gamma$ & Gaussian noise & SVD & Singular Value Decomposition \\
\hline$\sigma$ & Standard deviation & NWP & Numerical Weather Prediction \\
\hline
\end{tabular}

Tucker decomposition is an effective data-driven approach that can be used in many areas [14-18], such as image and signal processing, computer vision, data mining and fluid science. Here, we improve our previous wind field reconstruction work by using the Tucker decomposition technique.

In this paper, the authors propose a wind forecasting method by reconstructing the wind velocity distribution in a short time. The consideration is that if the wind field is much larger than the wind farm that is inside the wind field, then a certain period is required for the wind to travel from the border to the wind farm. The wind velocity in the wind farm is related to the wind speed from the boarder of the wind field, and it can be obtained before the wind reaches the wind farm. This is in fact equivalent to a short term wind forecast, and this period of time is the forecast time. This establishes the relationship between the wind field reconstruction and wind forecast for a wind farm situated inside the wind field.

In this paper a tensor-based method which combines a CFD database with a higher-order singular value decomposition method and the Least Square method is developed to improve the wind field reconstruction in 3-D spaces. The newly developed mathematical model is applied to CFD models with different geometric configurations. The reconstruction results are analysed at three elevations of the models. In addition, the new method is compared with the existing wind velocity distribution reconstruction method, and some interesting results are revealed. A range of parameters, e.g. sampling rate, noise error, sensor distribution as well as the number of sensors, are also taken into consideration so as to analyse their impact on the reconstruction error. Finally, wind tunnel experiments are carried out to test the performance of the proposed method. The experimental results also show that the newly proposed method has a better performance compared with the existing published method, leading to around $0.03 \%-0.4 \%$ lower relative reconstruction errors and $0.02 \%-1.7 \%$ lower maximum reconstruction errors, in most calculated cases.

\section{CFD database}

The data used in this paper are illustrated on a 3-D database, obtained from FLUENT with a series of inlet boundary conditions. This paper is concerned with steady flows passing an obstruction at a constant temperature. The motion of a compressible, Newtonian fluid is typically represented by mass and momentum (NavierStokes) conservation equations. The mass conservation is given by $\frac{\partial\left(\rho u_{x}\right)}{\partial x}+\frac{\partial\left(\rho u_{y}\right)}{\partial y}+\frac{\partial\left(\rho u_{z}\right)}{\partial z}=0$

where $\rho$ is the density $\left(\mathrm{kg} / \mathrm{m}^{3}\right)$, and $\mathrm{u}_{\mathrm{x}}, \mathrm{u}_{\mathrm{y}}, \mathrm{u}_{\mathrm{z}}$ represents velocity components $(\mathrm{m} / \mathrm{s})$ in the $\mathrm{x}, \mathrm{y}$ and $\mathrm{z}$ directions, respectively. The momentum equations are shown in equations (2)-(4).

$$
\begin{aligned}
& \nabla \bullet\left(\rho u_{x} \vec{u}\right)=-\frac{\partial p}{\partial x}+\frac{\partial \tau_{x x}}{\partial x}+\frac{\partial \tau_{y x}}{\partial y}+\frac{\partial \tau_{z x}}{\partial z}+\rho f_{x}, \\
& \nabla \bullet\left(\rho u_{y} \vec{u}\right)=-\frac{\partial p}{\partial y}+\frac{\partial \tau_{x y}}{\partial x}+\frac{\partial \tau_{y y}}{\partial y}+\frac{\partial \tau_{z y}}{\partial z}+\rho f_{y} \\
& \nabla \bullet\left(\rho u_{z} \vec{u}\right)=-\frac{\partial p}{\partial z}+\frac{\partial \tau_{x z}}{\partial x}+\frac{\partial \tau_{y z}}{\partial y}+\frac{\partial \tau_{z z}}{\partial z}+\rho f_{z} .
\end{aligned}
$$

Here $\tau$ is the shear stress, caused by the viscous effects, p represents the pressure $(\mathrm{Pa})$ and $\mathrm{f}$ represents the body forces on the fluid element $\left(\mathrm{m} / \mathrm{s}^{2}\right)$.

Based on equations (1)-(4), a CFD database is built. To obtain the CFD database, the inlet velocity is set to vary from 1 to $31 \mathrm{~m} / \mathrm{s}$ at $5 \mathrm{~m} / \mathrm{s}$ increments, and the inlet velocity direction changes from $0^{\circ}$ to $80^{\circ}$ with $20^{\circ}$ increments. The CFD database is also called the sample tensor $\mathrm{V}$, the dimension of which is $\mathrm{n} \times 4 \times \mathrm{m}$, where $\mathrm{n}$ is the number of nodes in a 2-D plane, and $m$ represents the number of sample matrices (which are also called snapshot matrices).

\section{Tensor and Tucker decomposition}

First of all, we briefly recall standard decomposition methods of matrices and tensors. Singular Value Decomposition was initially derived for square matrices by Beltrami in 1873; Eckart and Young applied SVD to rectangular matrices in 1939 [19], as shown in equation (5)

$\mathbf{V}=\mathbf{W} \Sigma \mathbf{U}^{\mathbf{T}}$

where matrix $\mathrm{V} \varepsilon \mathrm{R}^{\mathrm{n} \times \mathrm{m}}, \Sigma \varepsilon \mathrm{R}^{\mathrm{n} \times \mathrm{m}}, \mathrm{W} \varepsilon \mathrm{R}^{\mathrm{n} \times \mathrm{n}}$ and $\mathrm{U} \varepsilon \mathrm{R}^{\mathrm{m} \times \mathrm{m}}$.

$\Sigma=\left[\begin{array}{l}\Sigma_{1} \mathrm{O} \\ \mathrm{OO}\end{array}\right], \quad \Sigma_{1}=\operatorname{diag}\left(\sigma_{1}, \sigma_{2}, \ldots, \sigma_{\mathrm{r}}\right)$

In equation (6), $\sigma_{1} \geq \sigma_{2} \geq \ldots \geq \sigma_{\mathrm{r}}>0, \mathrm{r}=\operatorname{rank}(\mathrm{V})$. Normally, the first $\mathrm{k}$ columns of $\mathrm{W}$ can be used as basis vectors, which contain the 
main information of the CFD database.

When $V \varepsilon \mathrm{R}_{1}^{\mathrm{I} \times \mathrm{I} \times \mathrm{I}}{ }_{3}, \mathrm{~V}$ is a third-order tensor. In order to explore the main characters of tensor $\mathrm{V}$, the concept of tensor decomposition was originally introduced by Hitchcock in 1927 [20]. The Tucker decomposition can be explained in equation (7):

$\mathbf{V}=\mathbf{S} \times{ }_{1} \mathbf{U}^{(\mathbf{1})} \times{ }_{2} \mathbf{U}^{(\mathbf{2})} \times{ }_{3} \mathbf{U}^{(3)} \Rightarrow \mathbf{S}=\mathbf{V} \times{ }_{1} \mathbf{U}^{(\mathbf{1}) \mathbf{T}} \times{ }_{2} \mathbf{U}^{(\mathbf{2}) \mathbf{T}} \times{ }_{3} \mathbf{U}^{(\mathbf{3}) \mathbf{T}}$,

where $S \varepsilon \mathrm{R}_{1}^{\mathrm{J} \times \mathrm{J} \times \mathrm{J}_{3}}$ is the core tensor, $\mathrm{U}^{(1)} \varepsilon \mathrm{R}_{1}^{\mathrm{I} \times \mathrm{J}}{ }_{1}$ is a mode- 1 factorization factor matrix, $\mathrm{U}^{(2)} \varepsilon \mathrm{R}_{2}^{\mathrm{I} \times \mathrm{J}}{ }_{2}$ is a mode-2 factorization factor matrix and $\mathrm{U}^{(3)} \varepsilon \mathrm{R}_{3}^{\mathrm{I} \times \mathrm{J}_{3}}$ is a mode-3 factorization factor matrix. Fig. 1 shows the routine to decompose a three-way array tensor using the HOSVD method. It can be seen from equation (7) that the elements of the concerned tensor are calculated together and the element position is not changed.

Kolda introduced a method in 2006 [21,22] to represent an Nthorder tensor by a mode-n matrix $\mathrm{V}_{(\mathrm{n})}$, which can be called the Kolda unfolding. Fig. 2 shows the horizontal unfolding of a third-order tensor. When a third-order tensor is decomposed, the Kolda unfolding can be employed to obtain the tensor factorization factor matrices.

The following equation shows a way to calculate the mode-n factorization factor $\mathrm{U}^{(\mathrm{n})}$,

$\mathrm{V}_{(\mathrm{n})}=\mathrm{U}^{(\mathrm{n})} \Sigma \mathrm{P}^{(\mathrm{n}) \mathrm{T}}$.

\section{Mathematical description of the new method}

To reconstruct the wind velocity distributions in a short time, a new mathematical method based on tensor analysis is proposed in this section. Firstly, the measured wind velocity distribution data are stored in matrix $Y_{(m \times 4)}$, each column of which represents the absolute velocity, and velocity components $\mathrm{u}_{\mathrm{x}}, \mathrm{u}_{\mathrm{y}}$ and $\mathrm{u}_{\mathrm{z}}$, respectively. The relationship between $\mathrm{Y}$ and $\mathrm{X}$ can be shown as

$\mathbf{Y}=\mathbf{A X}$

where $X_{(n \times 4)}$ is the original wind velocity distribution matrix and $A_{(m \times n)}$ is the position matrix given by

$\mathbf{A}_{(\mathrm{i}, \mathrm{j})}=\left\{\begin{array}{ll}0, & \text { if the data is missing in the } \mathrm{i}-\text { th position } \\ 1, & \text { if the data is not missing in the } \mathrm{i}-\text { th position }\end{array}\right.$.
Normally, the number $m$ is much smaller than $n$, so it is difficult to solve equation (9). To overcome this difficulty, a new method was introduced to reduce the dimension of $\mathrm{X}$, which is now called the Tucker decomposition or higher-order singular value decomposition. We consider that the reconstructed matrix $X_{R}$ can be represented in a dimension-reduced manner, which takes the form of

$\mathbf{X}_{\mathbf{R}(:, \mathbf{i})}=\mathbf{W}_{(:, \mathbf{i},:)} \mathbf{B}_{(:, i)}$,

where the dimensions of $W, B$ and $X_{R}$ are respectively $n \times 4 \times k$, $\mathrm{k} \times 4$ and $\mathrm{n} \times 4$. In equation (11), $X_{R}$ represents the reconstructed result of the wind velocity distribution, and the tensor basis can be obtained from the Tucker decomposition, where $\mathrm{W}$ is determined by

$\mathbf{W}=\mathbf{S} \times{ }_{1} \mathbf{U}^{(\mathbf{1})} \times{ }_{2} \mathbf{U}^{(\mathbf{2})}$.

Considering the $i$-th column of Y, equation (9) can be rewritten as

$\mathbf{Y}_{(:, \mathbf{i})}+\gamma_{(:, \mathbf{i})} \approx \mathbf{A W}_{(:, \mathbf{i}::)} \mathbf{B}_{(:, \mathbf{i})}$,

where Gaussian noise $\gamma$ is introduced during the reconstruction process. The proposed noise $\gamma$ obeys a Normal distribution $N\left(0, \sigma^{2}\right)$ shown as

$\gamma(:, \mathrm{i}) \sim N\left(0, \sigma^{2}\right)$

where $\sigma$ represents the standard deviation of Gaussian noise. Now, the inverse problem of equation (9) is to solve the following equation

$\min f_{1}=\frac{1}{2}\|\mathbf{Y}(:, \mathbf{i})-\mathbf{A W}(:, \mathbf{i},:) \mathbf{B}(:, \mathbf{i})+\gamma(:, \mathbf{i})\|_{2}^{2}$,

and the coefficient matrix $B$ can be determined by the least squares method. After matrix B is known, the reconstruction result $X_{R}$ can be obtained by equation (11). The relative reconstruction error can be calculated by

Relative reconstruction error $(\mathrm{i})=\frac{\left\|\mathbf{X}_{\mathbf{R}(:, \mathrm{i})}-\mathbf{X}_{(:, \mathrm{i})}\right\|_{1}}{\left\|\mathbf{X}_{(:, \mathrm{i})}\right\|_{1}} \times 100 \%, \mathrm{i}$

$=1,2,3,4$.
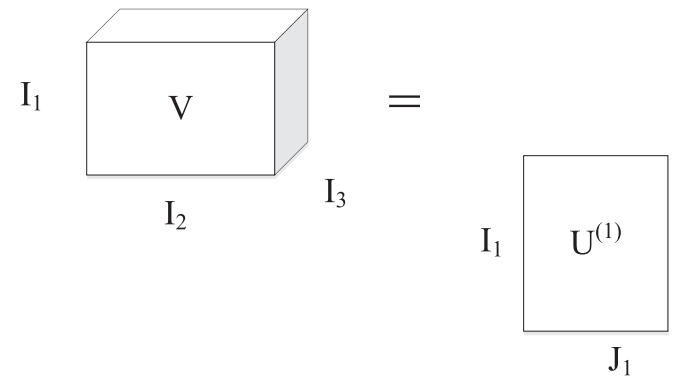
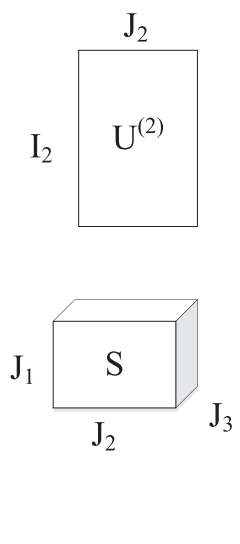

Fig. 1. HOSVD decomposition scheme. 


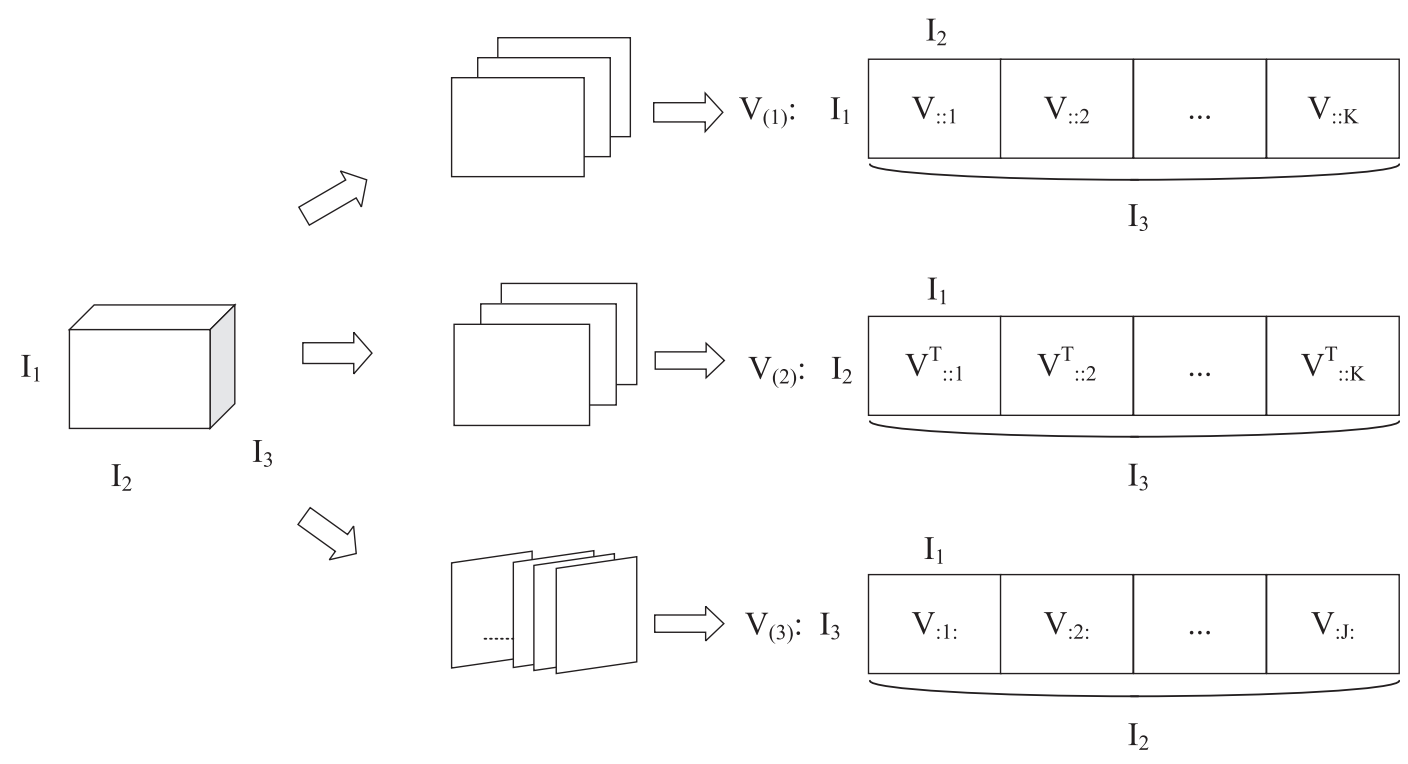

Fig. 2. Horizontal unfolding of three-way array (Kolda unfolding).

\section{Simulations}

The first step for reconstructing the wind velocity distribution is to set up a CFD database from various simulation cases. It is very important to choose appropriate geometry models to test the accuracy of the proposed mathematical model. In the current paper, three different types of CFD physical models are presented.

\subsection{Physical models in CFD}

Fig. 3 shows detailed geometric configurations of three Physical models tested in CFD simulations. The complexity of the models changes with the number of obstructions and their relative positions.

Table 1 shows that the height of the isolated ellipsoidal model is $0.15 \mathrm{~m}$ with the center at $(0,0,0)$. Meanwhile, two types of double sinusoidal models are installed, where the height of sinusoidal $A$ is $0.2 \mathrm{~m}$ and the height of sinusoidal $\mathrm{B}$ is $0.15 \mathrm{~m}$, and the distance between the center bases ( $A$ and $B$ ) is $0.5 \mathrm{~m}$. For the complex model, ellipsoid $A$, semi-sphere $B$ and sinusoidal $C$ are combined, and their heights are $0.15 \mathrm{~m}, 0.18 \mathrm{~m}$ and $0.2 \mathrm{~m}$, respectively. It should be noted that the ellipsoid used in the combined model is identical to the simple model with a long axis of $0.581 \mathrm{~m}$ and a short axis of $0.387 \mathrm{~m}$. The same analysis domain $(4 \mathrm{~m} \times 3 \mathrm{~m} \times 1 \mathrm{~m})$ is used for the three models.

ANSYS ICEM 16.0 is used for modelling. A mesh independency check is done, and the total mesh nodes of these three models are set to 238452,230944 and 137065 , respectively. The simulated results are obtained at several inlet boundary conditions. Specific planes are chosen to test the accuracy of the proposed method. ANSYS WORKBENCH FLUENT 16.0 is introduced to conduct a series of simulations, where the same standard $\mathrm{k}-\varepsilon$ turbulence model is used with the 2nd upwind difference scheme and standard wall functions. In this paper $\mathrm{k}-\varepsilon$ turbulence model is selected, due to the fact that in this study the physical models in the simulation are relatively simple with rather smooth changes of the shapes, thus k$\varepsilon$ model is quite often used in such or similar situations [23]. The model constants are set to ANSYS FLUENT 16.0 default values with an initial gauge pressure of 0 . The 'SIMPLE' solution method is used, the Gradient is set to 'Least Squares Cell Based' and the Pressure is set to 'Second Order' with initial gauge pressure. In this paper, the residual convergence of all simulations is set to $1 \times 10^{-5}$, and the calculation is set to 'steady'.

\subsection{Reconstruction results}

The reconstruction results of three different models are given respectively in sections 5.2.1, 5.2.2 and 5.2.3.

\subsubsection{Ellipsoidal model}

For the ellipse model, the velocity data in the plane at height $0.2 \mathrm{~m}$ is introduced here. The dimension of the sample tensor $\mathrm{V}$ is $10000 \times 4 \times 35$. Fig. 4 shows the information of the relative reconstruction errors when the number of tensor bases changes from 1 to 35 and the number of proposed sampling nodes is set to 100 with a Gaussian noise of standard deviation 0.001. It can be seen from Fig. 4 that the relative reconstruction error decreases with an increasing number of tensor bases. Especially for velocity $\mathrm{v}$, the relative reconstruction error (the maximum error is $18.9976 \%$ ) is higher than that of other directions. This is possibly due to the velocity in the $z$ direction being much smaller than in the other two directions. It can also be seen that when the number of tensor bases is more than 5 , the relative reconstruction errors in $\mathrm{x}, \mathrm{y}$ directions are nearly kept at $0.03 \%$, and the relative reconstruction error in $\mathrm{z}$ direction is kept at $0.88 \%$. It should be noted that in Fig. $4 \mathrm{u}$-velocity, $\mathrm{v}$-velocity and $\mathrm{w}$-velocity represent wind velocities in the $\mathrm{x}, \mathrm{z}, \mathrm{y}$ directions respectively.

Fig. 5 compares the results between the reconstructed streamlines and the CFD streamlines at a $0.2 \mathrm{~m}$ high plane. The number of the sampling nodes is set to 100 and the proposed standard deviation of Gaussian noise is set to 0.001 . Here, $\mathrm{u}, \mathrm{v}, \mathrm{w}$ represents the velocity in $\mathrm{x}, \mathrm{z}, \mathrm{y}$ direction, respectively. As a result, the overall relative reconstruction error is $0.0543 \%$, and the relative reconstruction error of $\mathrm{u}, \mathrm{v}, \mathrm{w}$ is $0.0462 \%, 1.1627 \%$ and $0.0697 \%$, respectively. Fig. 5 (a) gives the information about the reconstructed result and the CFD result of velocity $\mathrm{u}$ and velocity $\mathrm{w}$, where the reconstructed streamlines match the CFD streamlines well, so the red streamlines nearly overlap the black streamlines. Fig. 5 (b) shows that the reconstructed streamlines also match well with the CFD streamlines of velocity $\mathrm{u}$ and velocity $\mathrm{v}$, where velocity $\mathrm{v}$ is presented in the y direction. In Fig. 5 (c), the reconstructed streamlines and the CFD streamlines shown for velocity $\mathrm{v}$ and velocity $\mathrm{w}$ give $\mathrm{a}$ 


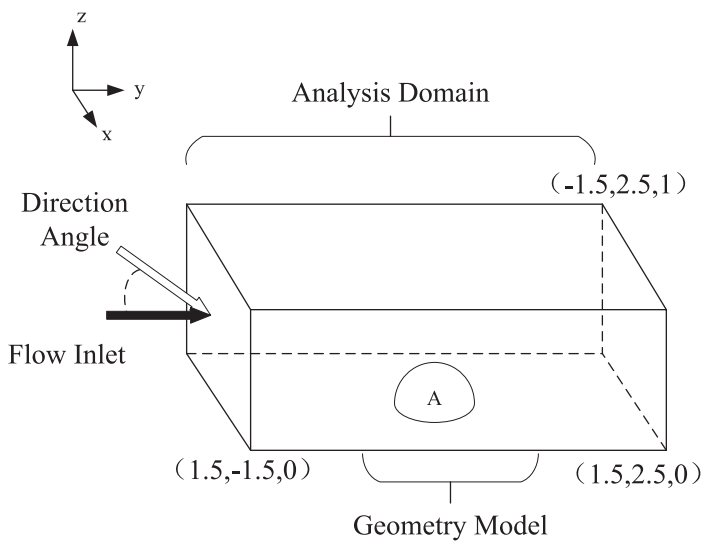

(a) 3-D geometry (Semi-Ellipsoid)

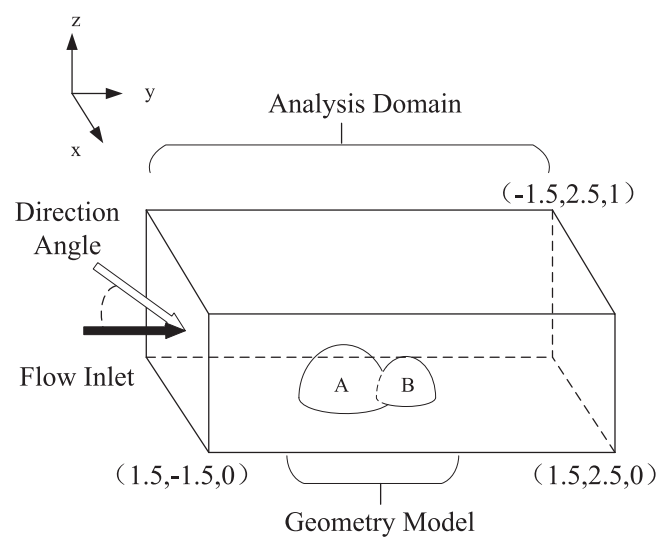

(b) 3-D geometry (Double sinusoidal model)

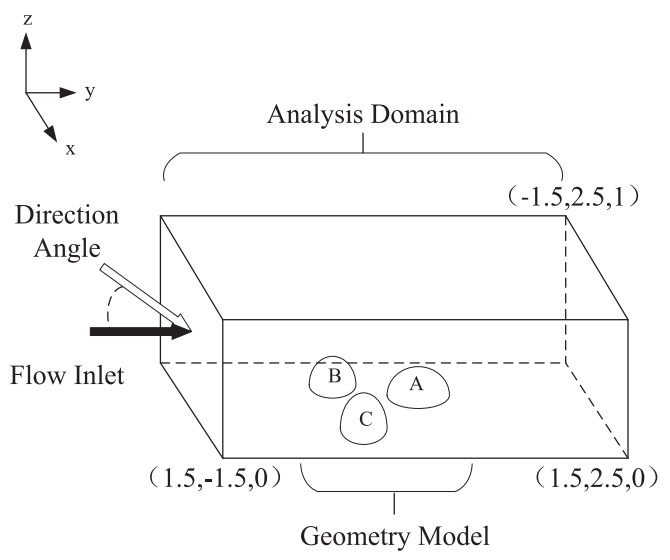

(c) 3-D geometry (Complex model)

Fig. 3. The proposed CFD geometry models and analysis domain.

Table 1

The detailed information of geometry.

\begin{tabular}{llll}
\hline & Geometry & Height $(\mathrm{m})$ & Bottom Center $(\mathrm{m})$ \\
\hline Ellipsoidal model & Semi-Ellipsoid-A & 0.15 & $(0,0,0)$ \\
Double sinusoidal model & Sinusoidal-A & 0.2 & $(0,0,0)$ \\
& Sinusoidal-B & 0.15 & $(0,0.5,0)$ \\
Complex model & Semi-Ellipsoid-A & 0.15 & $(0,0.3,0)$ \\
& Semi-Sphere-B & 0.18 & $(-0.3,-0.21,0)$ \\
& Sinusoidal-C & 0.2 & $(0.3,-0.21,0)$ \\
\hline
\end{tabular}

slightly larger difference compared with Fig. 5 (a)\&(b). It should be noted that velocity $v$ in Fig. 5 (c) is shown in the $x$ direction. In order to make the red lines clearer, Fig. 5(d)-5(f) represent the local amplification details of Fig. 5(a)-5(c), respectively.

\subsubsection{Double sinusoidal model}

The velocity data in the plane $(0.8 \mathrm{~m} \times 1.2 \mathrm{~m})$ at height $0.2 \mathrm{~m}$ for the double sinusoidal model is introduced here. The dimension of the sample tensor $\mathrm{V}$ is $9600 \times 4 \times 35$. Fig. 6 gives the change of relative reconstruction errors with the number of tensor bases rising from 1 to 35 . The number of proposed sampling nodes is set to 100 and a Gaussian noise with a standard deviation of 0.001 is introduced into the proposed mathematical model. It can be seen that Fig. 6 shows the same trend as Fig. 4. The largest error of velocity $\mathrm{v}$ is $16.3895 \%$, dropping to $0.3892 \%$ when the number of tensor bases is larger than 5 . The relative reconstruction errors of absolute velocity, velocity u and velocity w nearly drop from $1.9 \%$ to

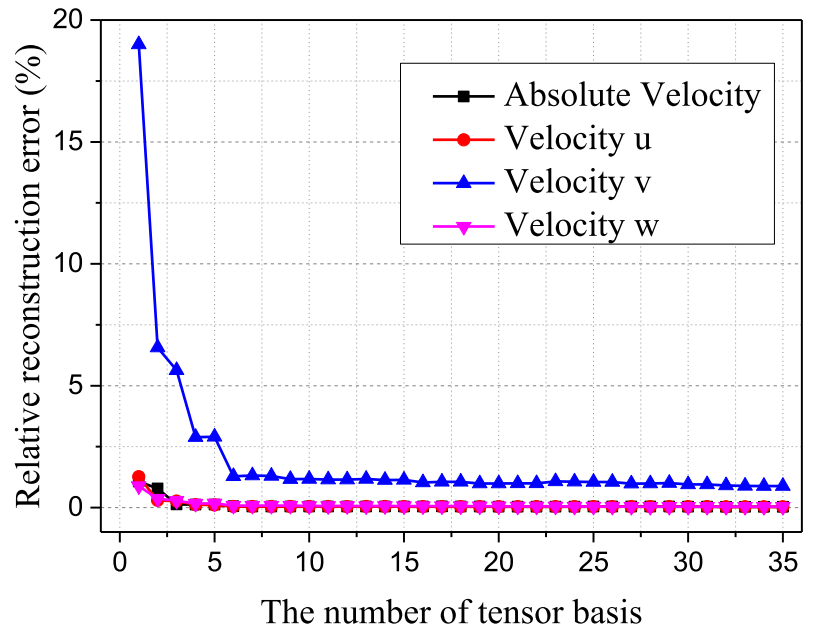

Fig. 4. Relative reconstruction errors with the increasing number of tensor basis (Semi-ellipsoid).

0.06\%. It should be noted that in Fig. 6 u-velocity, v-velocity and wvelocity represent wind velocities in $\mathrm{x}, \mathrm{z}, \mathrm{y}$ directions respectively.

Fig. 7 gives the reconstruction result of wind streamlines as well as CFD simulated streamlines in the plane $(0.8 \mathrm{~m} \times 1.2 \mathrm{~m})$ at the height of $0.2 \mathrm{~m}$, where the overall relative reconstruction error is $0.0914 \%$, and the relative reconstruction error of $\mathrm{u}, \mathrm{v}, \mathrm{w}$ is $0.1748 \%$, 


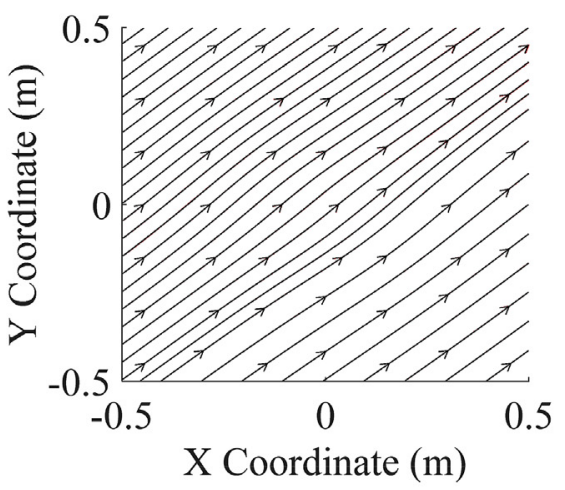

(a) Reconstructed streamlines and CFD streamlines showing velocity $\mathrm{u}$ and velocity $\mathrm{w}$

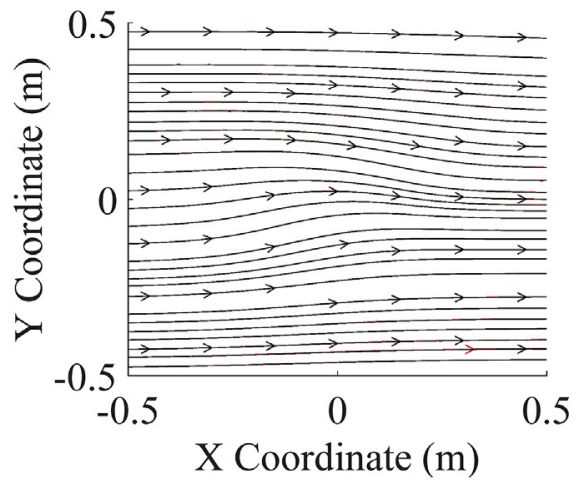

(b) Reconstructed streamlines and CFD streamlines showing velocity $\mathrm{u}$ and velocity $\mathrm{v}$

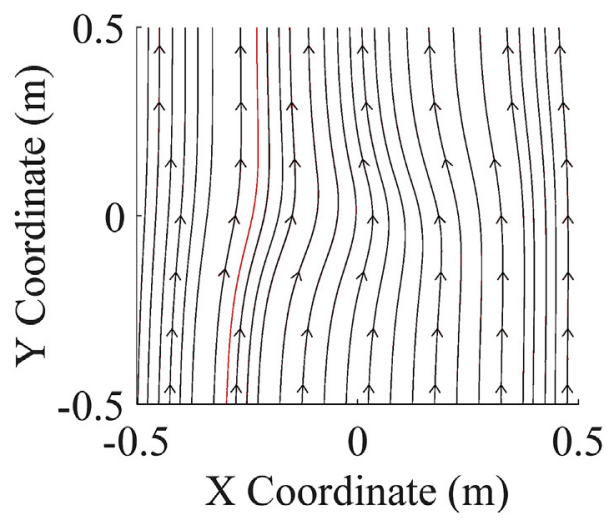

(c) Reconstructed streamlines and CFD streamlines showing velocity $\mathrm{v}$ and velocity $\mathrm{w}$

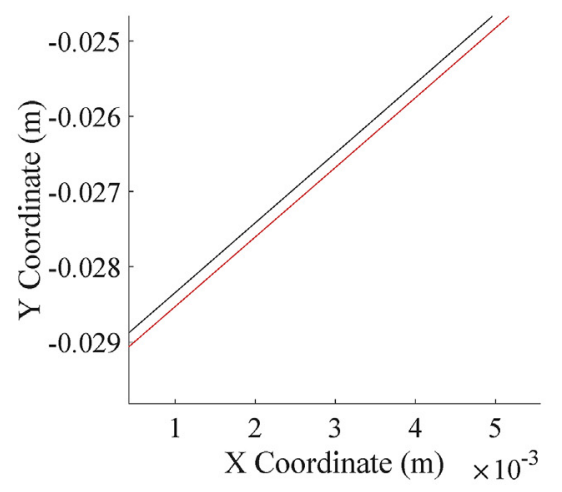

(d) The local amplification of figure 5.(a)

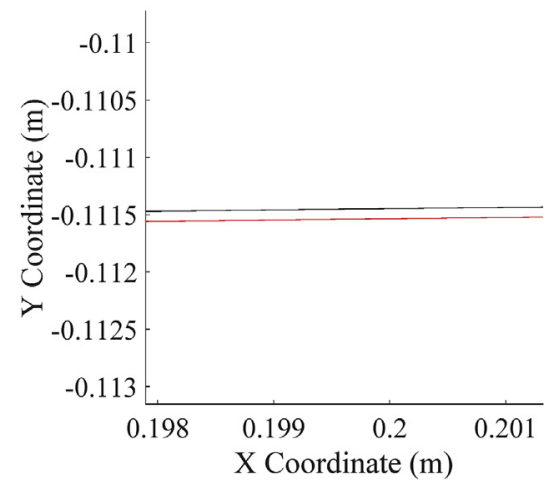

(e) The local amplification of figure 5.(b)

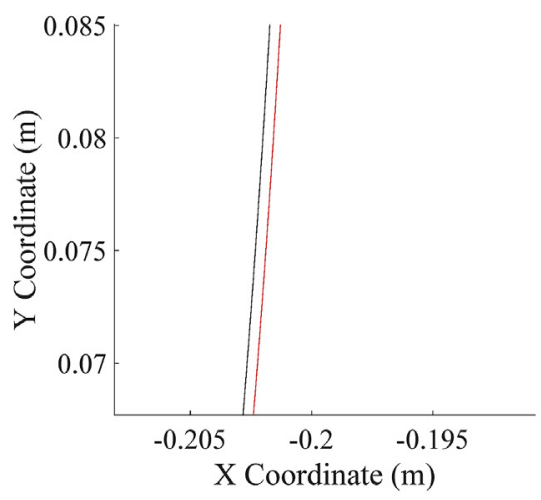

(f) The local amplification of figure 5.(c)

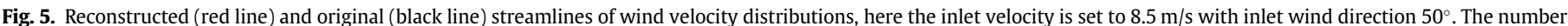

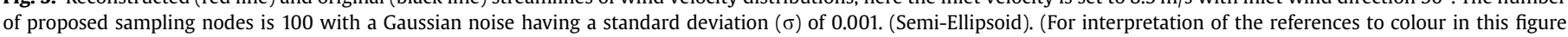
legend, the reader is referred to the Web version of this article.) 


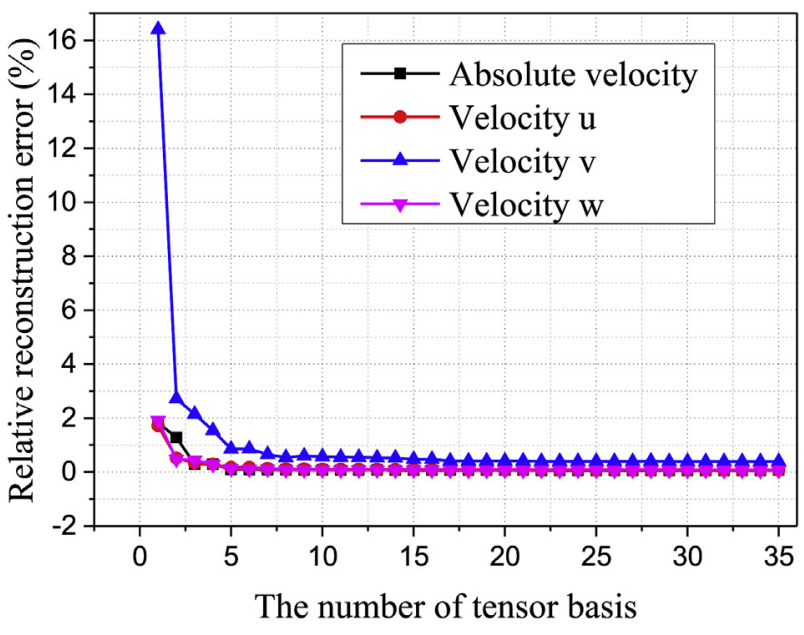

Fig. 6. Relative reconstruction errors with the increasing number of tensor bases (Double sinusoidal model).
$0.8633 \%$ and $0.1183 \%$. It can also be seen from Fig. 7 that the reconstructed result matches well with the original simulation result.

\subsubsection{Complex geometry model}

To test the accuracy of the proposed mathematical model, a complex physical model is introduced in this section. It can be seen from Fig. 8 that the relative reconstruction error of velocity $\mathrm{u}, \mathrm{v}, \mathrm{w}$ and the absolute velocity decreases when the number of the proposed tensor basis increases. Especially, when the tensor basis number is bigger than 5 , the relative error of absolute velocity drops to around $0.46 \%$. It is noted that in Fig. 8 u-velocity, v-velocity and $\mathrm{w}$-velocity represent wind velocities in $\mathrm{x}, \mathrm{z}, \mathrm{y}$ directions respectively.

Fig. 9 shows a particular case with the tensor bases number of 6 , and the sampling nodes number set to 100 with a Gaussian noise of standard deviation 0.001. Fig. 9 (a) compares the reconstructed streamlines and CFD streamlines in velocity $\mathrm{u}$ and velocity $\mathrm{w}$, and they match well. Same results are also shown in Fig. 9 (b) and (c). Overall, the relative reconstruction errors in the $\mathrm{x}, \mathrm{y}, \mathrm{z}$ directions are $0.4675 \%, 6.7230 \%$ and $0.7550 \%$, respectively.

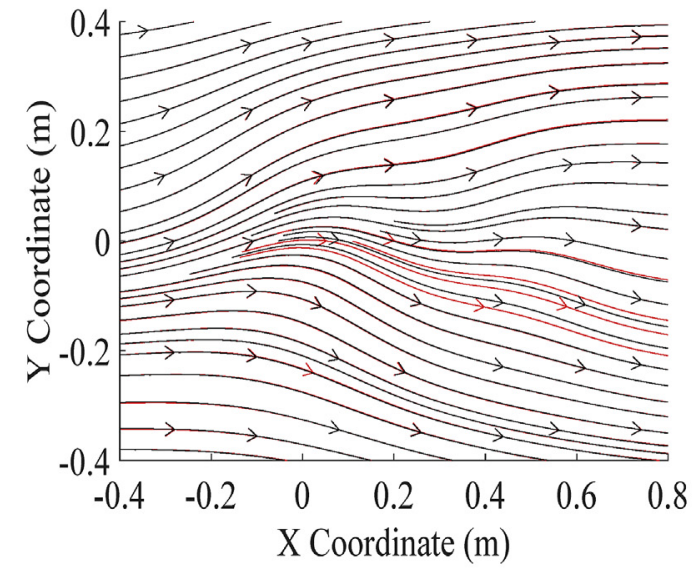

(b) Reconstructed streamlines and CFD streamlines showing velocity $\mathrm{u}$ and velocity $\mathrm{v}$ velocity $u$ and velocity $\mathrm{w}$

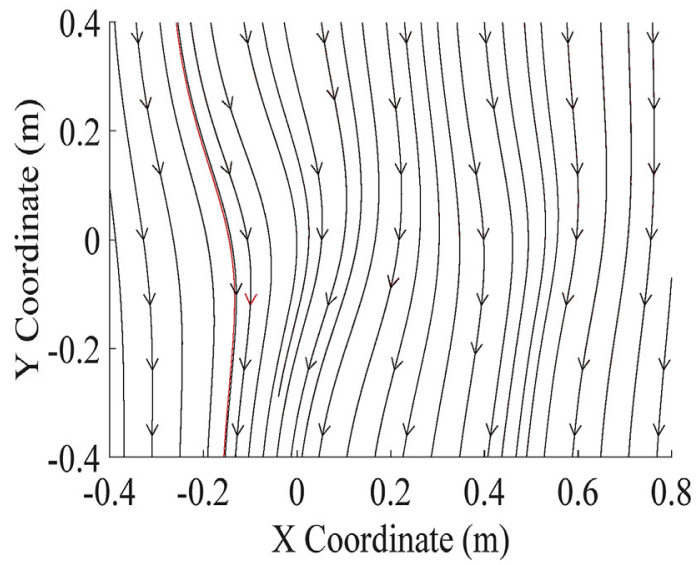

(c) Reconstructed streamlines and CFD streamlines showing velocity $\mathrm{v}$ and velocity $\mathrm{w}$

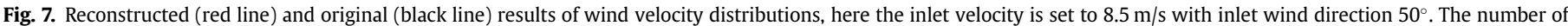

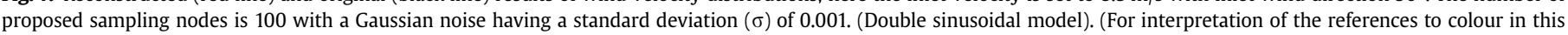
figure legend, the reader is referred to the Web version of this article.) 


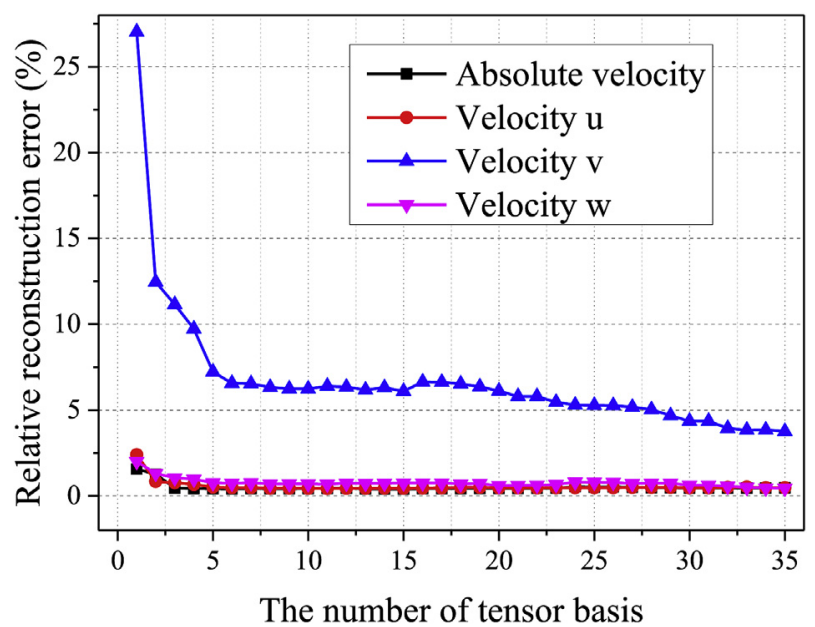

Fig. 8. Relative reconstruction errors with the increasing number of tensor basis (Complex model).

\subsection{Reconstruction results at different elevations}

Section 5.2 gives the calculation results in plane 1 at a height of $0.2 \mathrm{~m}$, so plane 2 (at a height of $0.22 \mathrm{~m}$ ) and plane 3 (at a height of

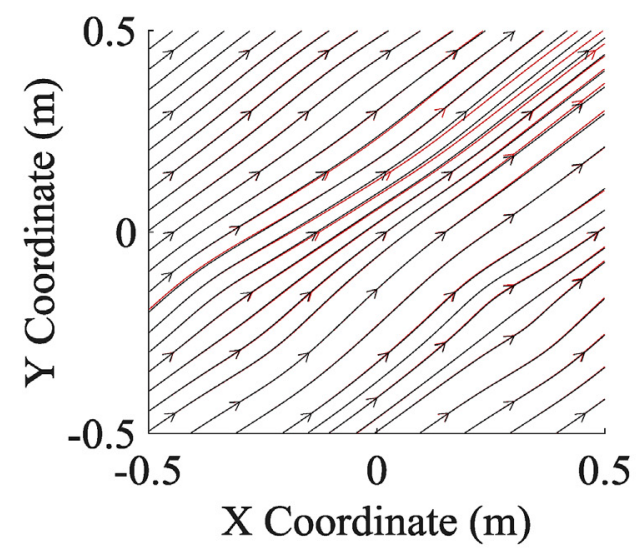

(a) Reconstructed streamlines and CFD streamlines showing velocity $\mathrm{u}$ and velocity $\mathrm{w}$
$0.24 \mathrm{~m}$ ) are also considered in this section in order to show more results and to test and validate the accuracy of the proposed method. More results in different planes are given in Fig. 10 for the complex geometry model. When the height is set to $0.22 \mathrm{~m}$, the overall relative reconstruction error is $0.2519 \%$, and the relative reconstruction error in velocity $\mathrm{u}, \mathrm{v}$, and $\mathrm{w}$ is $0.3764 \%, 5.7101 \%$ and $0.5297 \%$, respectively. Meanwhile, when the height is set to $0.24 \mathrm{~m}$, the overall relative reconstruction error is $0.1485 \%$, and the relative reconstruction error in velocity $\mathrm{u}, \mathrm{v}$, and $\mathrm{w}$ is $0.2979 \%, 5.2040 \%$ and $0.3757 \%$, respectively. These calculation results show a satisfactory validation.

\subsection{Error analysis}

Section 2 has shown satisfactory results of different types of geometry models, so the more complex geometry model is used for error analysis. Sampling rate, noise level as well as sensor distributions are considered in this section to give a detailed analysis of errors.

5.4.1. Sensitivity of reconstruction error on sampling rate

Fig. 11 (a) shows information about the relative reconstruction error of the absolute velocity based on 3 different sensor distributions. It can be seen that when the sampling rate is lower than a

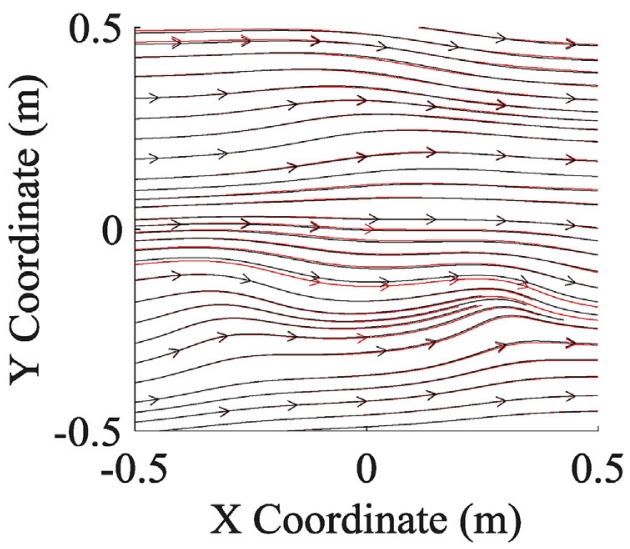

(b) Reconstructed streamlines and CFD streamlines showing velocity $u$ and velocity $v$

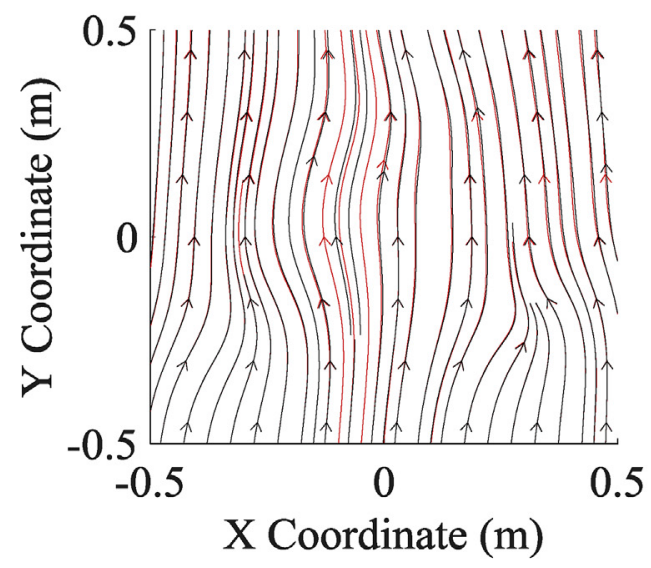

(c) Reconstructed streamlines and CFD streamlines showing velocity $\mathrm{v}$ and velocity $\mathrm{w}$

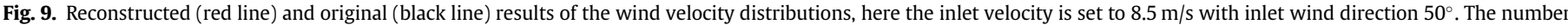

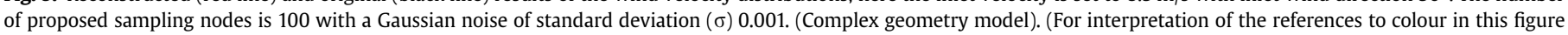
legend, the reader is referred to the Web version of this article.) 


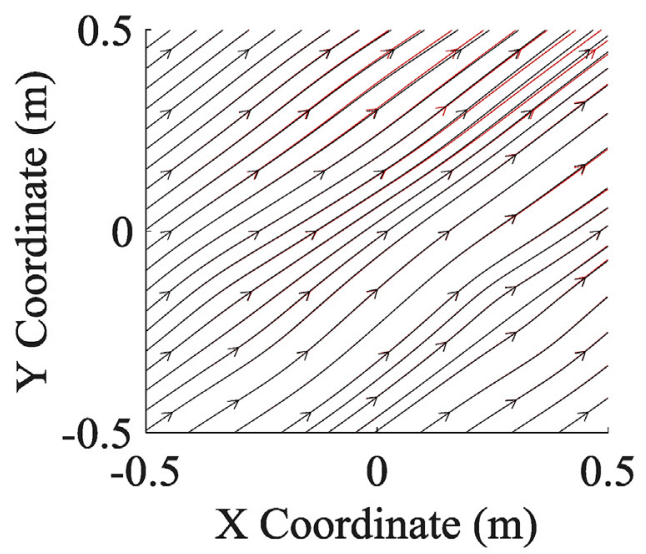

(a) Reconstructed streamlines and CFD streamlines showing velocity $u$ and velocity $w$ (plane 2 )

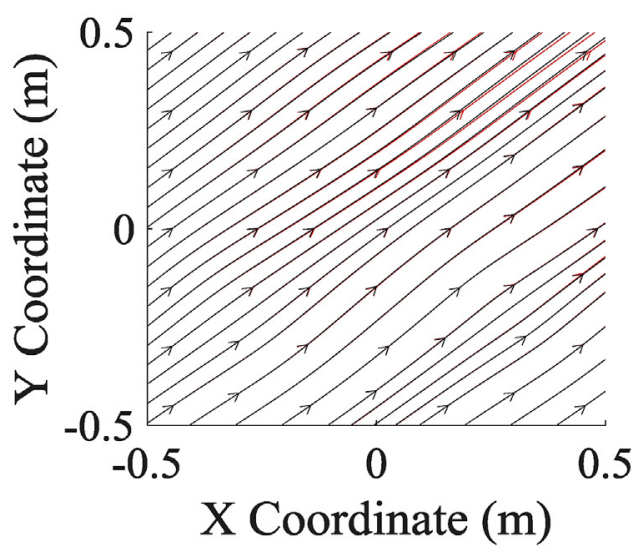

(b) Reconstructed streamlines and CFD streamlines showing velocity $u$ and velocity $w$ (plane 3 )

Fig. 10. Reconstructed result and CFD result shown in plane 2 and plane 3 (Complex geometry model).

specific figure (around $0.2 \%$ ), the relative reconstruction result can be influenced by the distribution of sensors. In addition, with an increasing sampling rate, the relative reconstruction error nearly stays at the same level. Fig. 11 (b)-(d) give a similar result compared with Fig. 11 (a).

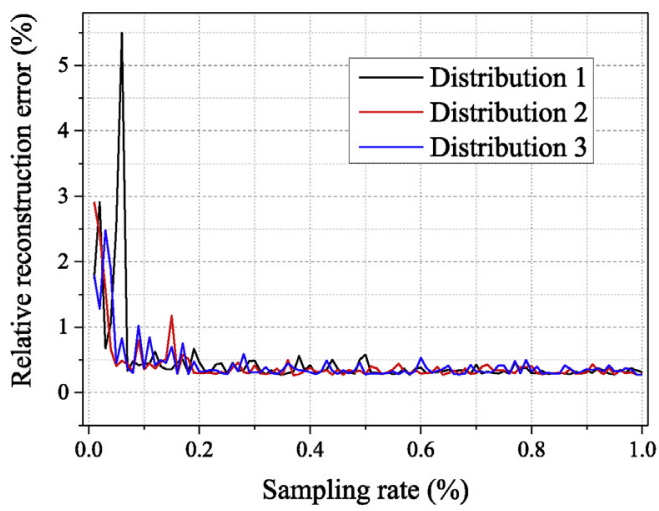

(a) The relative reconstruction error of absolute velocity based on 3 different sensor distributions

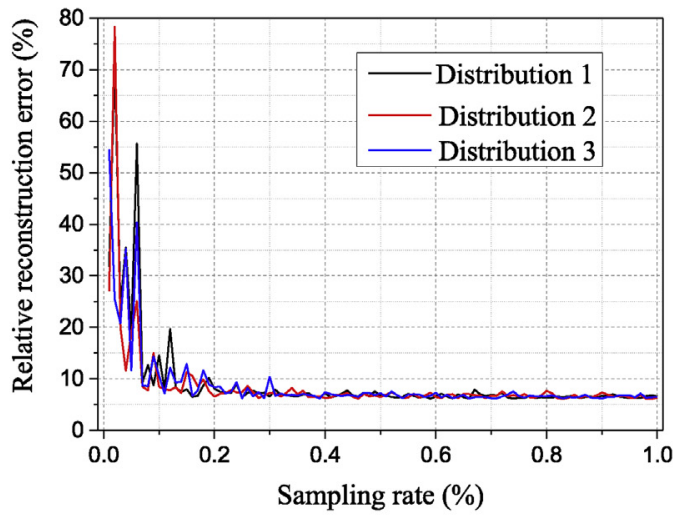

(c) The relative reconstruction error of velocity $\mathrm{v}$ based on 3 different sensor distributions

\subsubsection{Sensitivity of reconstruction error on noise level}

The first 2, 4, 6, 8, 10 tensor bases are proposed in this section with Gaussian noise of standard deviation from 0 to $20 \%$. Here, the noise level is represented by standard deviation. The targeted test case is the one with inlet velocity of $8.5 \mathrm{~m} / \mathrm{s}$ and inlet direction of $50^{\circ}$. Fig. 12 shows the relative reconstruction error of wind velocity

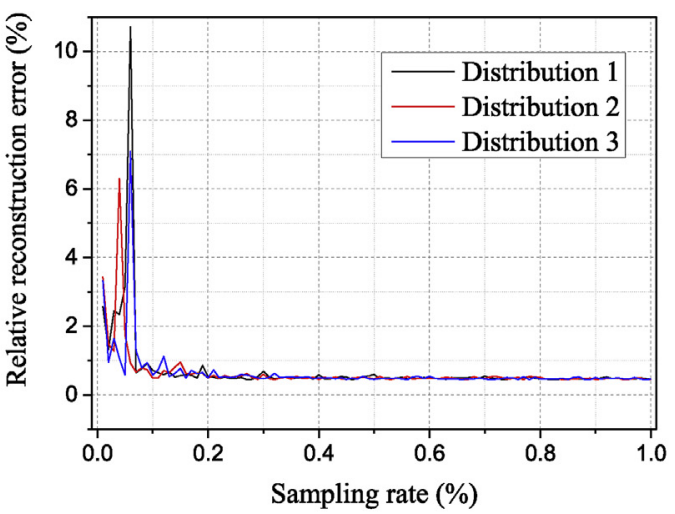

(b) The relative reconstruction error of velocity $u$ based on 3 different sensor distributions

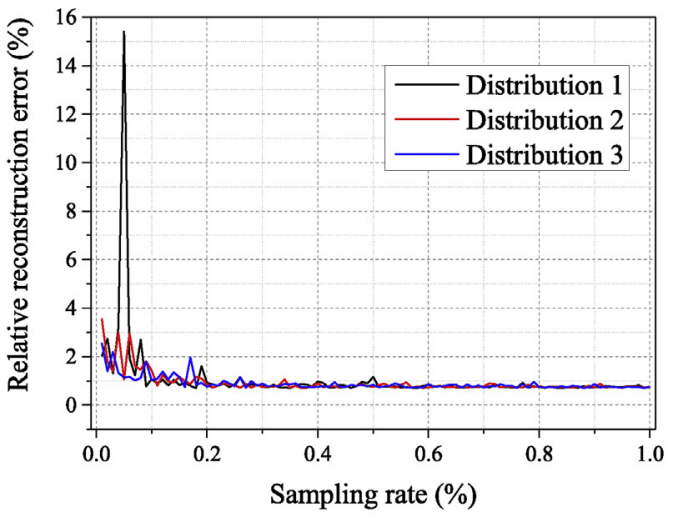

(d) The relative reconstruction error of velocity $w$ based on 3 different sensor distributions

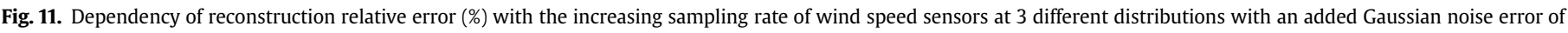
standard deviation $0.1 \%$. The inlet boundary conditions are set to $8.5 \mathrm{~m} / \mathrm{s}$ for inlet wind velocities with $50^{\circ}$ of inlet wind direction. 


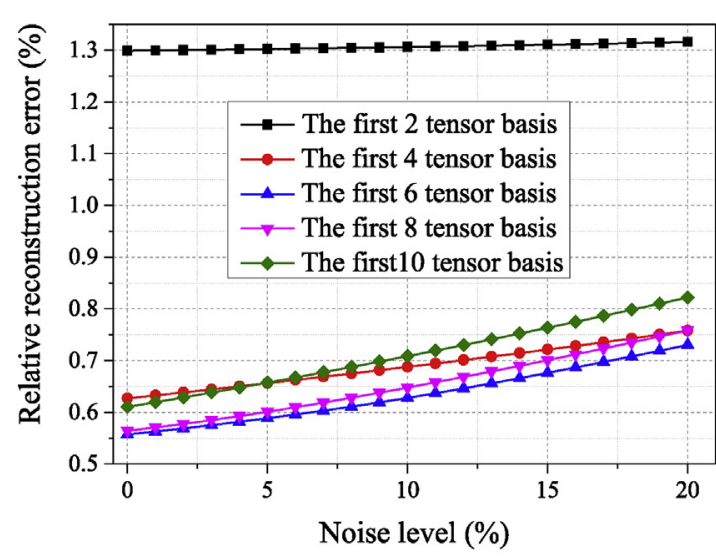

(a) The relative reconstruction error of absolute velocity based on different noise levels and the number of tensor bases

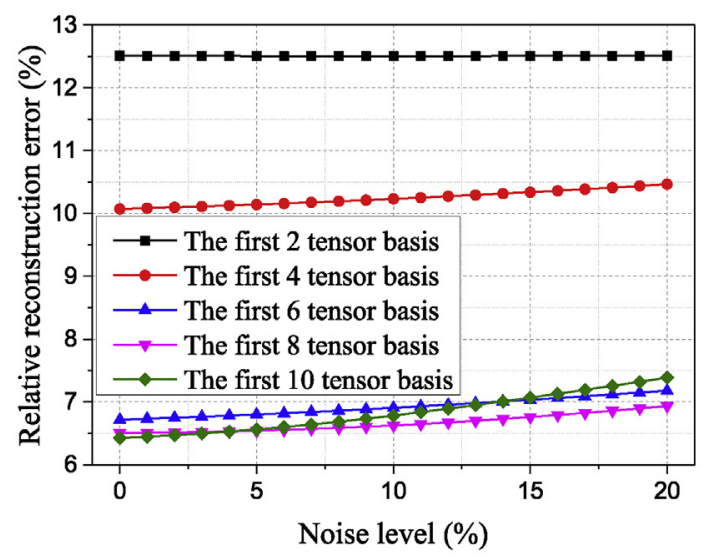

(c) The relative reconstruction error of velocity $v$ based on different noise levels and the number of tensor bases

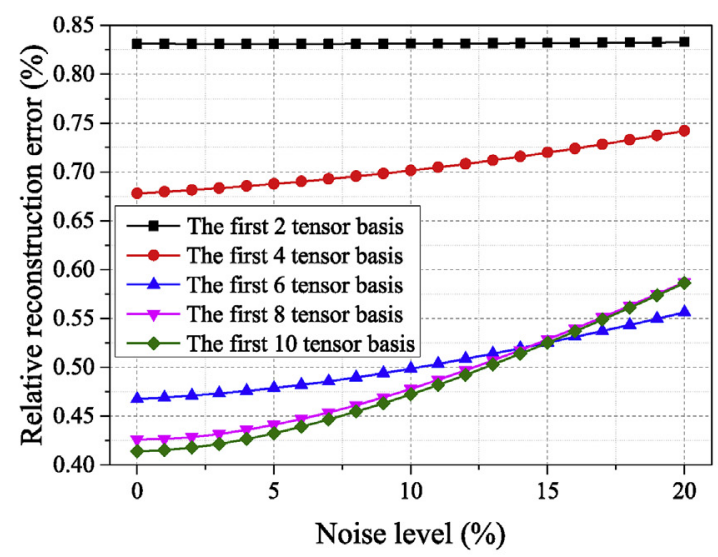

(b) The relative reconstruction error of velocity $u$ based on different noise levels and the number of tensor bases

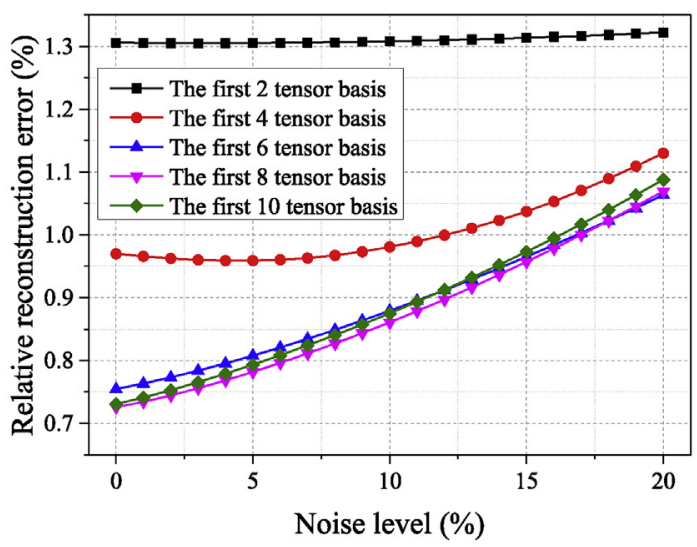

(d) The relative reconstruction error of velocity w based on different noise levels and the number of tensor bases

Fig. 12. Dependency of reconstruction error with the increasing noise level, the first 2, 4, 6, 8, 10 tensor bases are considered.

distributions in any direction, and a similar trend is given by Fig. 12 (a)\&(b)\&(c)\&(d), where the relative reconstruction error is more sensitive to the noise level as the number of the proposed tensor bases increases.

\subsection{Method comparison}

The authors have introduced a new method based on tensor analysis to reconstruct wind velocity distributions, which can be applied to higher dimensional data. In order to test the accuracy of the proposed method, it is compared with a previous method which employs a classic SVD technique. Fig. 13 shows the maximum error $(\mathrm{m} / \mathrm{s})$ calculated by the two methods in plane 1 at a height of $0.2 \mathrm{~m}$, and the maximum error in $i$-th column is calculated using equation (17):

$$
\begin{aligned}
\operatorname{Maximum} \operatorname{Error}(\mathbf{i}) & =\operatorname{Max}\left|\mathbf{X}_{\mathbf{R}(:, \mathrm{i}) \mathbf{j}}-\mathbf{X}_{(:, \mathrm{i}) \mathrm{j}}\right|, \quad \mathrm{i}=1,2,3,4 ; \mathbf{j} \\
& =1,2, \ldots, 10000 .
\end{aligned}
$$

In this paper, the authors mainly focus on the wind velocity distributions in any direction. Fig. 13 (b)-(d) shows that the proposed method has a smaller maximum error compared to the previous method, which means that the proposed method is more suitable to find the intrinsic relationship between the velocities from different directions, even though Fig. 13 (a) shows that when the number of the proposed bases is chosen between 10 and 20, a slightly higher error in the proposed method is found. Essentially the two methods yield comparable errors, with the new method showing slight improvement, Fig. 13(c) and (d).

In order to compare more results by more methods, the relative reconstruction errors are given in Fig. 13(e)-(h). With the number of proposed basis changing from 2 to 35 , it can be seen that the relative errors from the new method show a slight improvement over the previous method. The only exception is Fig. 13(f) where the number of proposed basis is 1 . However, this should not be a concern in real applications where a number of basis vectors will seldom be 1 , since the errors caused by such a choice will always be large, whichever methods used. Therefore, in general the new method is slightly better than the previous one. 


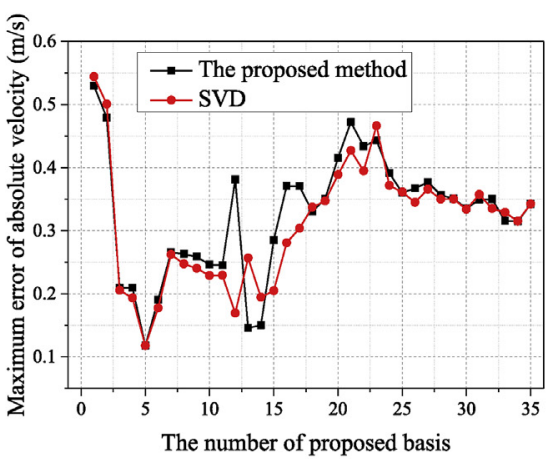

(a) The maximum errors of absolute velocity with the number of proposed basis changing from 1 to 35

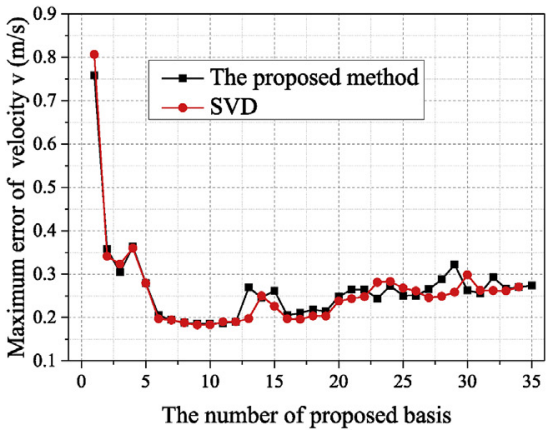

(c) The maximum errors of velocity $\mathrm{v}$ with the number of proposed basis changing from 1 to 35

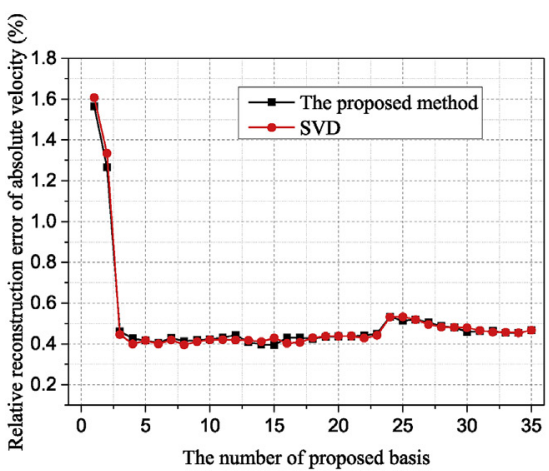

(e) The relative errors of absolute velocity with the number of proposed basis changing from 1 to 35

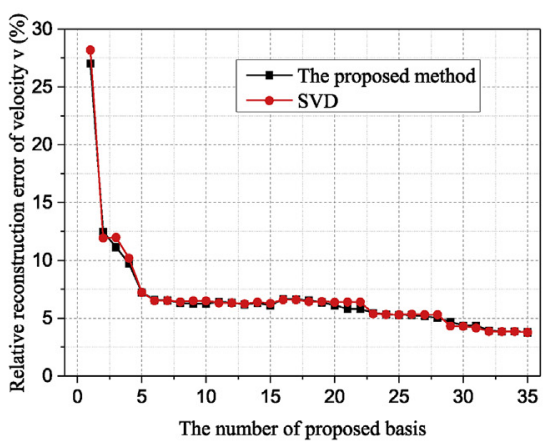

(g) The relative errors of velocity $\mathrm{v}$ with the number of proposed basis changing from 1 to 35

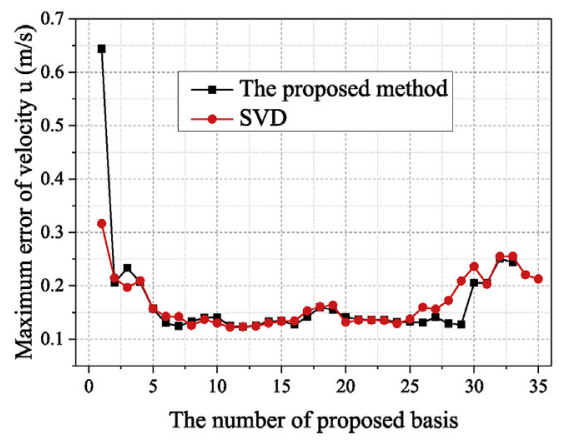

(b) The maximum errors of velocity $u$ with the number of proposed basis changing from 1 to 35

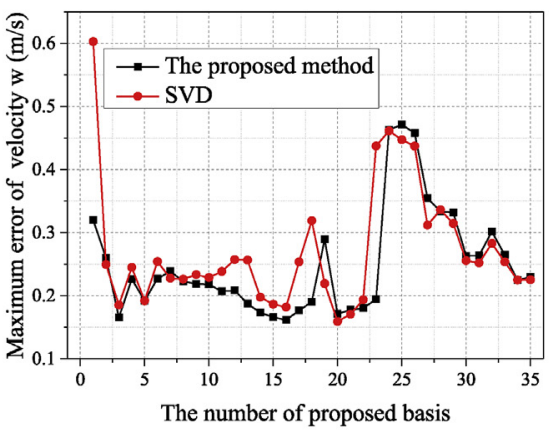

(d) The maximum errors of velocity $w$ with the number of proposed basis changing from 1 to 35

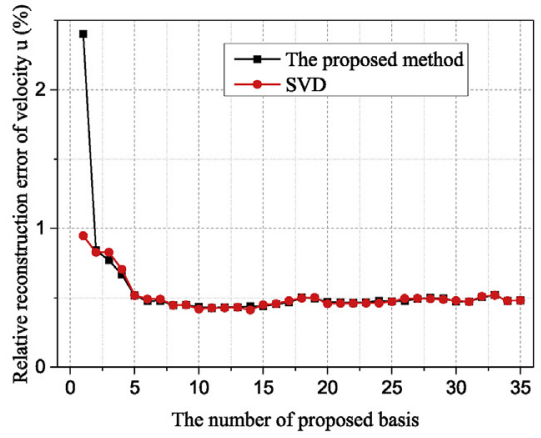

(f) The relative errors of velocity $u$ with the number of proposed basis changing from 1 to 35

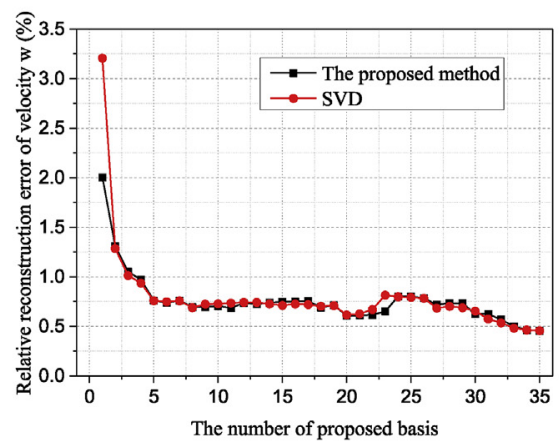

(h) The relative errors of velocity $w$ with the number of proposed basis changing from 1 to 35

Fig. 13. Comparison between the proposed method and the previous method. 


\section{Experimental validation}

A wind tunnel experiment is used in this section to test the accuracy of the proposed method, so the calculated result of the proposed algorithm is compared with the experimental result.

\subsection{Experimental setup}

The experimental facility consists of a wind tunnel $(11 \times 3 \times 1 \mathrm{~m})$ housing the obstruction, i.e. the physical models, in the test section, a fan and a data acquisition system (Fig. 14). A variable diameter section was used to connect the test section with the fan. Fig. 14 shows the test section of $4 \times 3 \times 1(\mathrm{~m})$, the transition section with guiding vanes to regulate the wind directions, and the stabilizing section of $1.6 \mathrm{~m}$ between the test section and transition section. Considering the transition section with a length of $3 \mathrm{~m}$, two guiding vanes are installed, and the length of the first blade is $1.2 \mathrm{~m}$. To obtain reliable wind tunnel results, a series of tests at each point are carried out, and then the average results are used as the measured data. According to [13], the precision of the sensor (hotwire anemometer) is $1.5 \%$ with the resolution of $0.1 \mathrm{~m} / \mathrm{s}$, and these could also support the reliability of this measured data. The complex geometry model was used as the test object, and wind speed sensors (hotwire anemometers) were placed at selected locations on a $9 \times 9$ grid around the test object. The sensors were connected to a data logger, in order to transfer the data to a computer. The guiding vanes (in the transition section) was used to regulate the wind speed in the test section.

Fig. 15 shows the complex geometry model that is used in this wind tunnel experiment. The data were collected from the plane at a height of $0.22 \mathrm{~m}$.

Here, each time wind speed sensors are placed at selected positions where they do not have too much influence on the original flow, as Fig. 16 shows.

The procedure of this experiment is given below:

1. All anemometers are calibrated and positioned at their grid locations.

2. The fan speed is adjusted by using a $45 \mathrm{~kW}$ inverter.

3. Wind speed data ( 120 values for each location) are acquired using the data acquisition system (over a time span of $3 \mathrm{~min}$ ). The average speeds are used for each point.

4. The fan is switched off and the anemometers are positioned at new locations.

5. The above procedure is repeated, until all the required grid locations are covered.

\subsection{Experimental results}

During the reconstruction process, the CFD database is built for the combined geometry model according to section 5. After
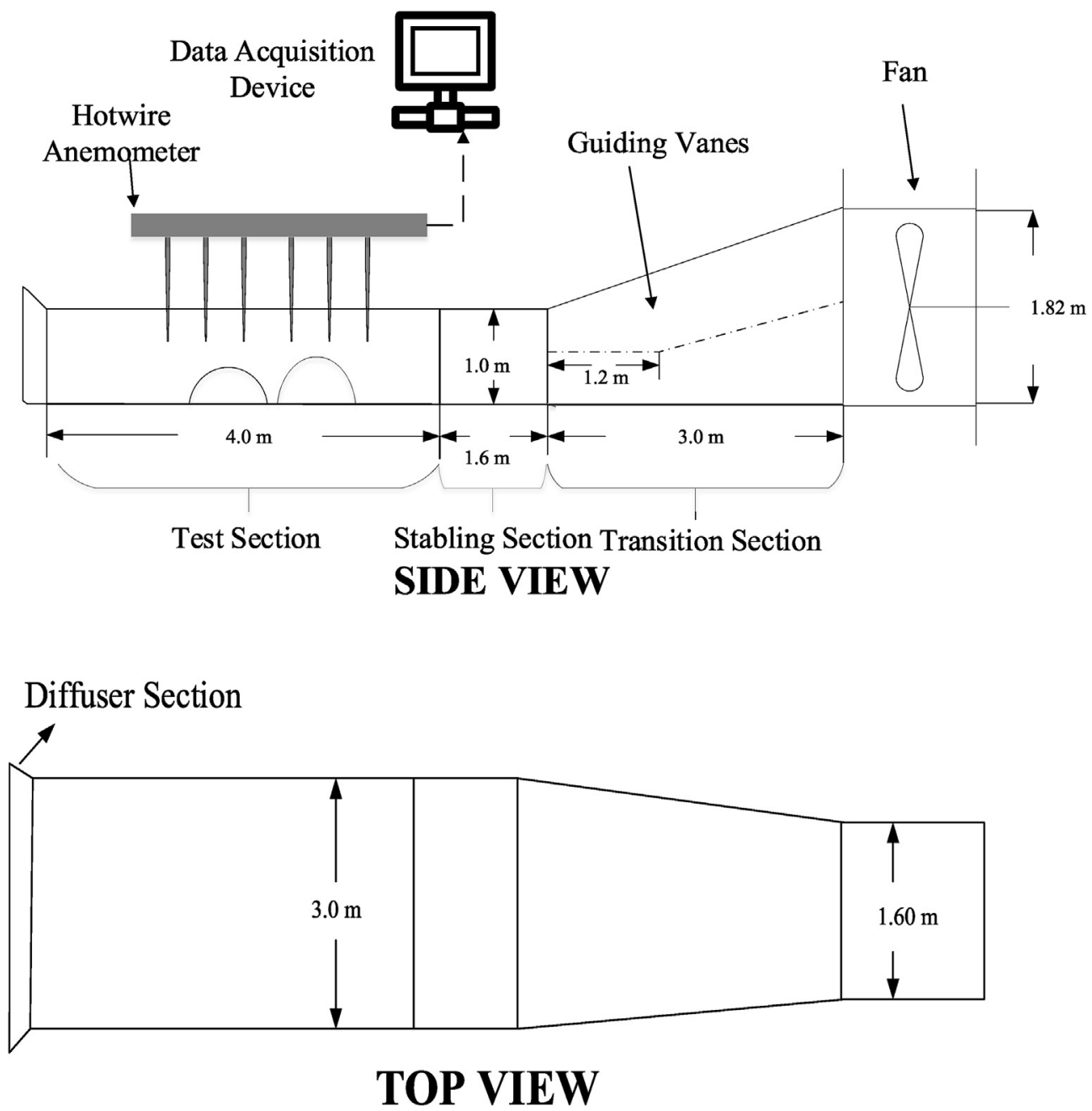

Fig. 14. Experiment setup. 


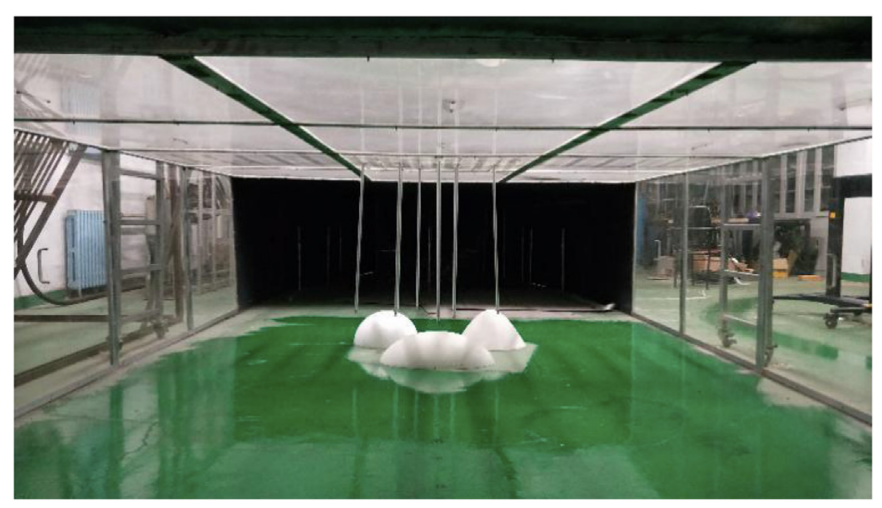

Fig. 15. Wind tunnel experiment in real time.

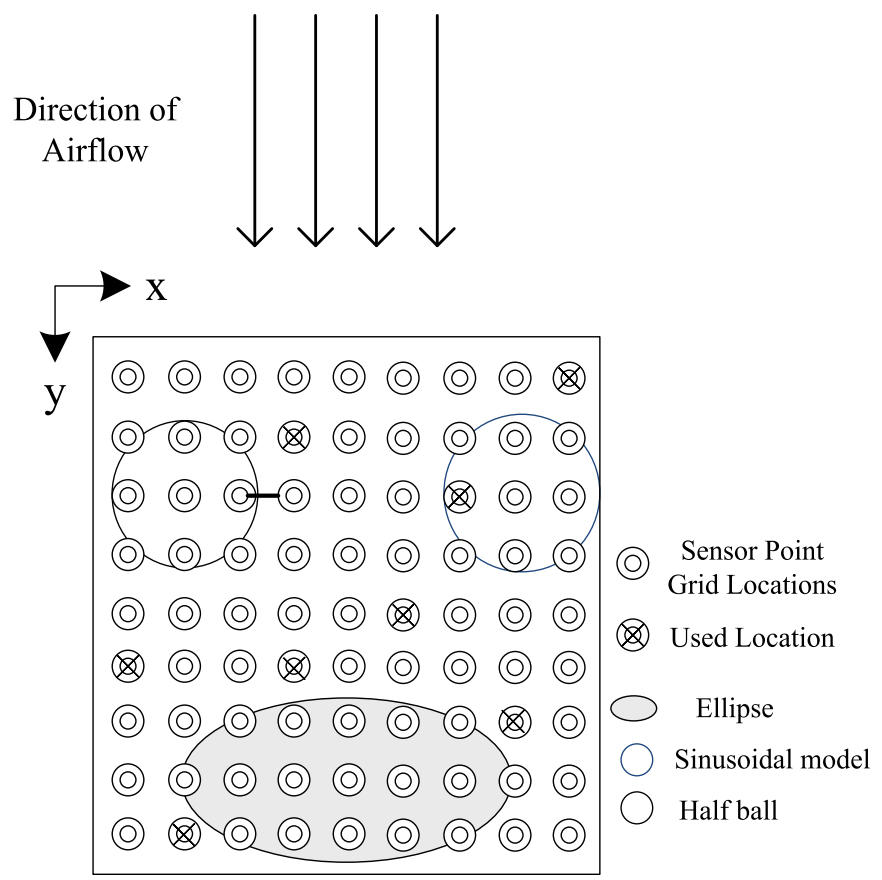

Fig. 16. Top view of the test part.

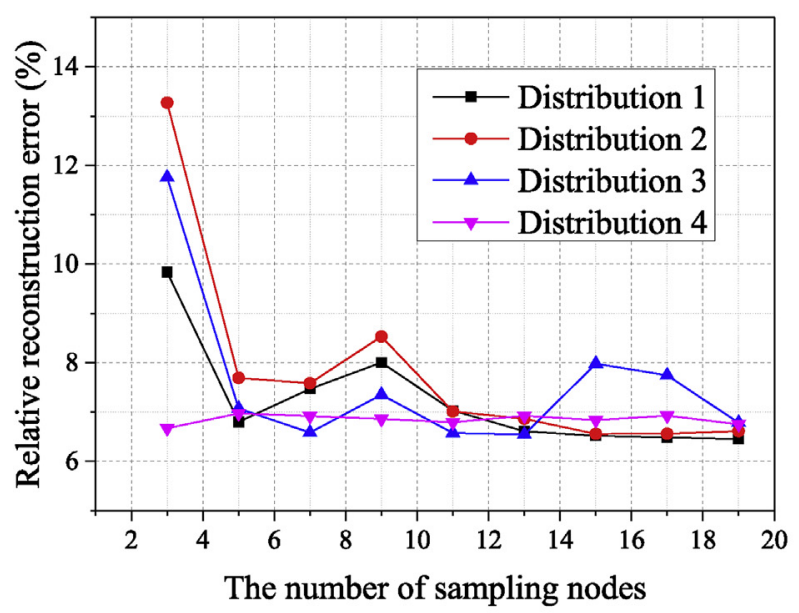

(a) The first 2 tensor bases obtaining tensor bases from the CFD database off site, sensor measured data are used to reconstruct the wind velocity distribution on site. Here, the number of proposed nodes is set to 3, 5, 7, 9, $11,13,15,17$ and 19 , and these nodes are placed at different places using the first 2 and 3 tensor bases. Fig. 17 (a) \& (b) give the result of four different sensor distributions with random combinations of the sensor placement. Even though the sensor distribution is changed, it can still be seen that the relative reconstruction errors generally do not exceed about $10 \%$. The relative error may generate from measurement data. In addition, the wind speed distribution in real time is more natural compared with the CFD database, the inlet velocity of which is set to uniform flow. From Fig. 17, we could also see that the sensor distribution has little influence on reconstruction error when the number of sampling nodes is larger than a certain value (around 10). For the same number of sampling nodes, there are four different combinations of sensor placement, shown in Figs. 17 and 18. Distribution 1-distribution 4 are in fact arranged at randomly picked locations.

Fig. 18 shows the relative reconstruction error of the proposed method with four sensor distributions (random combinations of sensor placement) with the number of tensor bases increasing from 1 to 12 , and the number of sampling nodes set to 16 . It can be seen from Fig. 18, when the tensor bases number is smaller than 6 , the reconstruction error is relatively smaller, and the sensor distribution also has little influence on the reconstruction error. However, when the number of tensor bases is larger than 6 , reconstruction errors become larger for all sensor distributions. It can be seen that the relative reconstruction errors nearly stay between $8 \%$ and $6 \%$ when the number of tensor bases increases from 1 to 6 , while the difference of relative reconstruction error (among these 4 distributions) becomes larger when the number of tensor bases is more than 6 . Thus, for the real time reconstruction process, the number of tensor bases is important for the reconstruction performance. Figs. 17 and 18 show that the proposed method has a good performance for experimental cases with a satisfactory reconstruction error. It can be seen from Fig. 18 that when the number of tensor bases is set to 6 in the experimental case, the relative reconstruction error is the lowest, so 6 tensor bases are suggested in this manuscript. In many cases we have studied we can see a general trend that the error curve tends to level off after several basis vectors have been used. Therefore normally a few bases, say 6 , can be an initial choice. When the geometry model in CFD is changed,

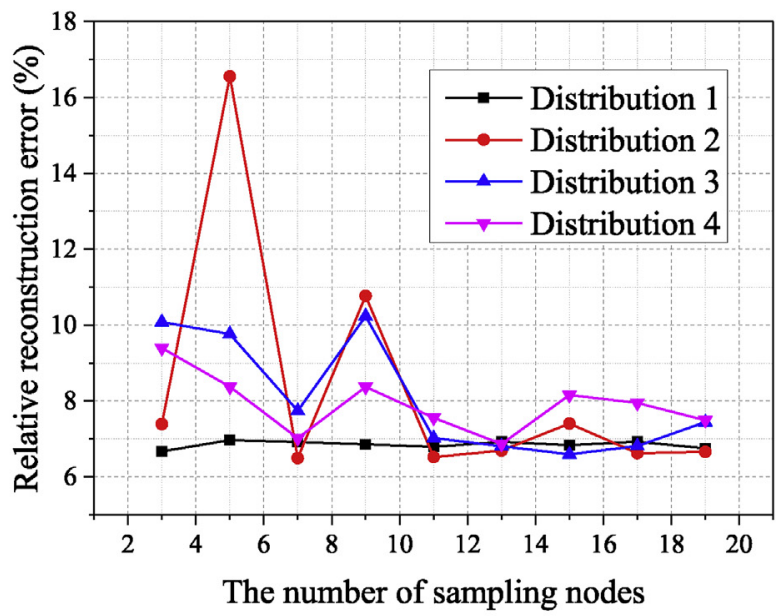

(b) The first 3 tensor bases

Fig. 17. Relative reconstruction error (\%) with different number of proposed nodes with 4 different distributions. 


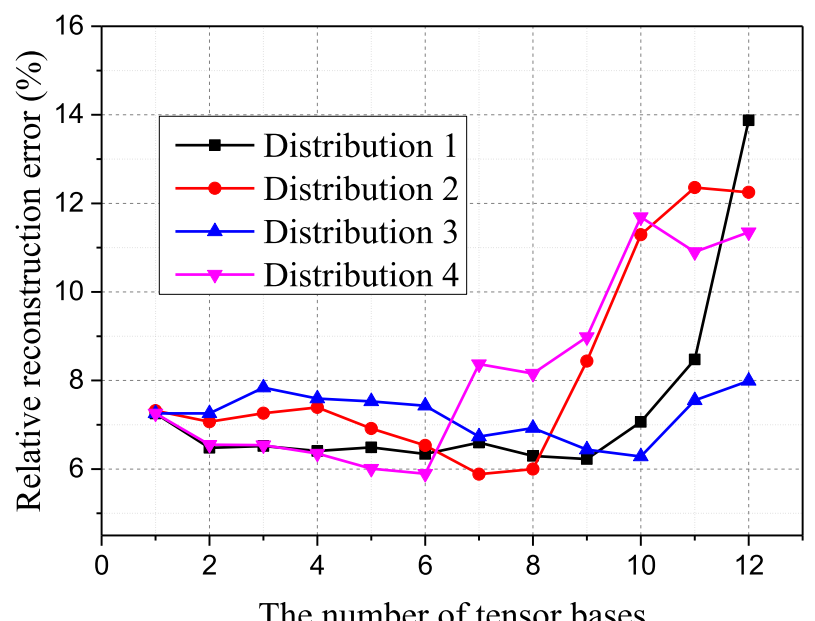

Fig. 18. Relative reconstruction error (\%) with different number of tensor bases at 4 different distributions.

more analyses will need be given.

In order to compare the performance between the newly proposed method and an existing method [9], a series of tests are carried out and the comparison results are given in Figs. 19 and 20, Tables 2-4. Fig. 19 shows wind velocity distributions, and a smaller difference between the reconstruction result of the proposed method (yellow bar) and sensor measured data (red bar) than the difference between the reconstruction of the proposed method and sensor measured data. To show the calculated results in Fig. 19 more clearly, Fig. 20 gives some representative comparison results, from which we can see that the height of red bar (the calculated results of the new proposed method) is closer to that of the yellow bar (sensor measured data).

It can be seen from Table 2 that when the first 3 bases are employed with the 6 sampling nodes, the maximum errors (at five

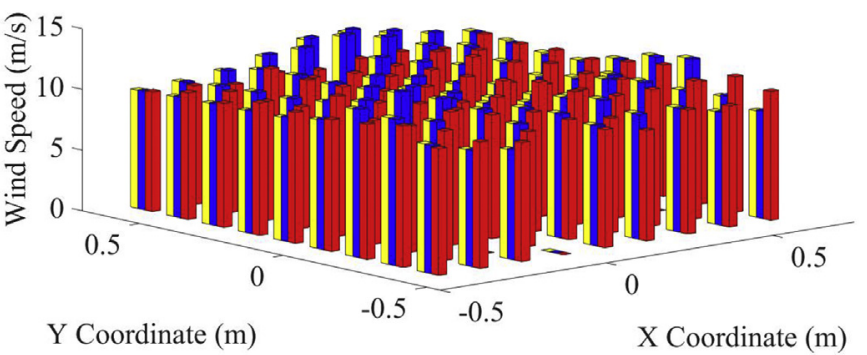

Fig. 19. Wind Velocity Distribution shown in experimental results (red bar), the reconstruction results using the proposed method (yellow bar) and the reconstruction results using the previous method (blue bar), the number of sampling nodes is set to 6 and the first 3 bases being used. (For interpretation of the references to colour in this figure legend, the reader is referred to the Web version of this article.)

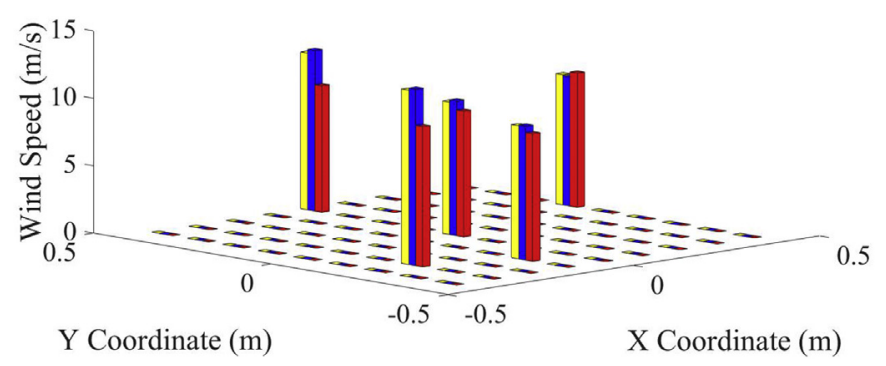

Fig. 20. Some representative points of Fig. 19.

random distributions) of the new tensor-based method are the lowest, and the proposed method also sees lower relative reconstruction errors at these distributions with $6.5454 \%, 7.7522 \%$, 8.0783\%, 6.6166\% and 6.6305\% respectively, and the figures for the existing method are 6.6255\%, 8.2876\%, 8.3073\%, 6.7148\% and $6.8733 \%$. When the number of the sampling nodes is set to 10 ,

Table 2

Comparison between the proposed method and the existing method, the number of sampling nodes is set to 6 and the first 3 bases being used.

\begin{tabular}{|c|c|c|c|c|c|}
\hline & Distribution 1 & Distribution 2 & Distribution 3 & Distribution 4 & Distribution 5 \\
\hline \multicolumn{6}{|l|}{ The proposed method } \\
\hline $\mathrm{RE}(\%)$ & 6.5454 & 7.7522 & 8.0783 & 6.6166 & 6.6305 \\
\hline Maximum error (\%) & 19.1782 & 28.5453 & 25.2029 & 18.4927 & 24.1383 \\
\hline \multicolumn{6}{|l|}{ The existing method } \\
\hline $\mathrm{RE}(\%)$ & 6.6255 & 8.2876 & 8.3073 & 6.7148 & 6.8733 \\
\hline Maximum error (\%) & 19.0334 & 31.0963 & 25.6753 & 17.7275 & 24.9878 \\
\hline
\end{tabular}

Table 3

Comparison between the proposed method and the existing method, the number of sampling nodes is set to 10 and the first 3 bases being used.

\begin{tabular}{|c|c|c|c|c|c|}
\hline & Distribution 1 & Distribution 2 & Distribution 3 & Distribution 4 & Distribution 5 \\
\hline \multicolumn{6}{|l|}{ The proposed method } \\
\hline $\mathrm{RE}(\%)$ & 9.1854 & 6.4618 & 6.4494 & 7.1403 & 6.817 \\
\hline Maximum error (\%) & 29.7943 & 21.4059 & 20.0256 & 25.1918 & 24.2729 \\
\hline \multicolumn{6}{|l|}{ The existing method } \\
\hline $\mathrm{RE}(\%)$ & 9.2191 & 6.5001 & 6.8068 & 7.2359 & 7.0781 \\
\hline Maximum error (\%) & 30.2314 & 21.1056 & 20.049 & 25.7244 & 25.9017 \\
\hline
\end{tabular}

Table 4

Comparison between the proposed method and the existing method, the number of sampling nodes is set to 14 and the first 3 bases being used.

\begin{tabular}{|c|c|c|c|c|c|}
\hline & Distribution 1 & Distribution 2 & Distribution 3 & Distribution 4 & Distribution 5 \\
\hline \multicolumn{6}{|l|}{ The proposed method } \\
\hline $\mathrm{RE}(\%)$ & 7.1231 & 6.8507 & 6.8107 & 7.7665 & 7.2041 \\
\hline Maximum error (\%) & 19.2448 & 25.5128 & 21.257 & 19,306 & 24.5673 \\
\hline \multicolumn{6}{|l|}{ The existing method } \\
\hline $\mathrm{RE}(\%)$ & 7.1295 & 6.9731 & 6.7982 & 8.1431 & 7.26 \\
\hline Maximum error (\%) & 19.4413 & 25.6607 & 21.5824 & 20.5414 & 24.6889 \\
\hline
\end{tabular}


Table 3 shows that the relative reconstruction error of the proposed method is lower compared with the figure for the existing method in most cases, and the relative reconstruction error nearly drops $0.03 \%-0.4 \%$. From Table 3, it can also be seen that in most cases, the maximum error of the proposed method is about $0.02 \%-1.7 \%$ lower than the figure for the existing method. In addition, there are also lower maximum errors and relative reconstruction errors of the proposed method in Table 4. Overall, compared with the existing method, the proposed method sees around 0.03\%-0.4\% lower relative reconstruction error and $0.02 \%-1.7 \%$ lower maximum reconstruction errors, in most cases.

\section{Conclusions}

In this paper, a higher-order singular value decomposition method and the least squares method (based on a CFD database and measured sensor data) have been successfully introduced to reconstruct wind velocity distributions in a short time. The proposed reconstruction method gives a new way for wind forecasting in 3-D space, and this research mainly shows that:

i. The proposed method can be used to solve this three-way array problem in a short time and the low reconstruction error shows the accuracy of this model.

ii. The proposed model can be applied to different-types of CFD geometry models at variable planes.

iii. Compared with the existing method, the new method shows a better result for wind velocity distributions. The experimental results show that the relative reconstruction errors calculated by the new proposed method are around $0.03 \%$ $0.4 \%$ lower than the figure for the existing method, and the maximum reconstruction errors calculated by the new proposed method are around $0.02 \%-1.7 \%$ lower than the figure for the existing method, in most cases.

iv. The sensor distribution does not have much influence on the reconstruction error, especially when the sampling rate is higher than a specific amount which is also small.

v. The proposed model shows a good performance for filtering noisy data.

vi. The reconstruction result of the proposed method matches well with the sensor measured data of a real experimental case.

Overall, this paper proposes a novel method to predict wind velocity distributions in 3-D. In the future, the authors will focus on time-series wind predictions. However, the understandings of more turbulent conditions, together with the complications in time and space, such as the topology of the wind field, will need more studies in the future, which is the aim of our future work.

\section{Acknowledgments}

The authors wish to extend their gratitude to NSFC for sponsoring the research (No 61571189, 61871181) and to the State Administration of Foreign Experts Affairs for supporting the 111 project (ref: B13009).

\section{References}

[1] Liu H, Wu H, Li Y. Smart wind speed forecasting using EWT decomposition, GWO evolutionary optimization, RELM learning and IEWT reconstruction. Energy Convers Manag 2018/04/01/2018;161:266-83.

[2] Hoolohan V, Tomlin AS, Cockerill T. Improved near surface wind speed predictions using Gaussian process regression combined with numerical weather predictions and observed meteorological data. Renew Energy 2018/10/ 01; $126: 1043-54$.

[3] Stathopoulos C, Kaperoni A, Galanis G, Kallos G. Wind power prediction based on numerical and statistical models. J Wind Eng Ind Aerod 2013/01/01;112: 25-38.

[4] Milanese M, Tornese L, Colangelo G, Laforgia D, de Risi A. Numerical method for wind energy analysis applied to Apulia Region, Italy. Energy 2017/06/ $01 ; 128: 1-10$

[5] De Giorgi MG, Ficarella A, Tarantino M. Assessment of the benefits of numerical weather predictions in wind power forecasting based on statistical methods. Energy 2011/07/01;36:3968-78.

[6] Khosravi A, Machado L, Nunes RO. Time-series prediction of wind speed using machine learning algorithms: a case study Osorio wind farm, Brazil. Appl Energy 8/15/2018;224:550-66.

[7] Wang C, Zhang H, Fan W, Ma P. A new chaotic time series hybrid prediction method of wind power based on EEMD-SE and full-parameters continued fraction. Energy 2017/11/01;138:977-90.

[8] Blonbou R. Very short-term wind power forecasting with neural networks and adaptive Bayesian learning. Renew Energy 2011/03/01;36:1118-24.

[9] Akçay H, Filik T. Short-term wind speed forecasting by spectral analysis from long-term observations with missing values. Appl Energy 2017/04/01;191: 653-62.

[10] Salcedo-Sanz S, Ortiz-García EG, Pérez-Bellido ÁM, Portilla-Figueras A, Prieto L. Short term wind speed prediction based on evolutionary support vector regression algorithms. Expert Syst Appl 2011/04/01;38:4052-7.

[11] Yang Z, Wang J. A hybrid forecasting approach applied in wind speed forecasting based on a data processing strategy and an optimized artificial intelligence algorithm. Energy 2018/10/01;160:87-100.

[12] Dong L, Wang L, Khahro SF, Gao S, Liao X. Wind power day-ahead prediction with cluster analysis of NWP. Renew Sustain Energy Rev 2016/07/01;60: 1206-12.

[13] Qin L, Liu S, Long T, Shahzad MA, Schlaberg HI, Yan SA. Wind field reconstruction using dimension-reduction of CFD data with experimental validation. Energy 2018/05/15;151:272-88.

[14] Luo X, Zhang Z, Zhang C, Wu X. Multi-focus image fusion using HOSVD and edge intensity. J Vis Commun Image Represent 2017/05/01;45:46-61.

[15] Zhang X, Xu Z, Jia N, Yang W, Feng Q, Chen W, et al. Denoising of 3D magnetic resonance images by using higher-order singular value decomposition. Med Image Anal 2015/01/01;19:75-86.

[16] Moreno AI, Jarzabek AA, Perales JM, Vega JM. Aerodynamic database reconstruction via gappy high order singular value decomposition. Aero Sci Technol 2016;52:115-28.

[17] Padhy S, Dandapat S. Validation of $\mu$-volt T-wave alternans analysis using multiscale analysis-by-synthesis and higher-order SVD. Biomed Signal Process Control 2018/02/01;40:171-9.

[18] Afra S, Gildin E. Tensor based geology preserving reservoir parameterization with higher order singular value decomposition (HOSVD). Comput Geosci 2016/09/01;94:110-20.

[19] Eckart C, Young G. A principal axis transformation for non-hermitian matrices. Bull Am Math Soc 1939;45:118-21.

[20] Hitchcock FL. The expression of a tensor or a polyadic as a sum of products. Stud Appl Math 1927;6:164-89.

[21] Kolda TG. Multilinear operators for higher-order decompositions. Sandia Report SAND2006-2081. Albuquerque, New Mexico and Livermore, California: Sandia National Laboratories; 2006.

[22] Kolda T, Bader B. The TOPHITS model for higher-order web link analysis. In: Workshop on link analysis, counterterrorism \& security; 2006.

[23] Li L, Liu Y-q, Yang Y-p, Han S, Wang Y-m. A physical approach of the shortterm wind power prediction based on CFD pre-calculated flow fields. J Hydrodynam Ser B 2013/02/01;25:56-61. 